\title{
The Role of Seawater and Saline Solutions in Treatment of Respiratory Conditions
}

\author{
Danijela Štanfel ${ }^{1 .}{ }^{*}$, Livije Kalogjera ${ }^{2}$, Sergej V. Ryazantsev ${ }^{3}$, Kristina Hlača ${ }^{{ }^{*}}$, Elena Y. Radtsig ${ }^{4}$, Rashidov Tei- \\ muraz ${ }^{5}$, Pero Hrabač ${ }^{6}$, Vasyl I. Popovich ${ }^{7}$
}

Jadran-Galenski Laboratorij d.d., Svilno 20, 51000 Rijeka, Croatia; danijela.stanfel@jgl.hr; kristina.hlaca@jgl.hr

2 Sisters of Mercy University Hospital, Department of Otorhinolaryngology/Head and Neck Surgery, Vinogradska 29, Zagreb, Croatia;_kalogjera@sfzg.hr

3 National State Research Institute of Ear, Throat, Nose and Speech, Bronnitskaya street, bld 9, S-Petersburg, Russia; professor.ryazantsev@mail.ru

4 Russian National State Pirogov Medical University, Ulitsa Ostrovityanova, 1, Moscow, Russia; radena@rambler.ru

5 Regional Center of Traumatology and Ortopedics Department of Otorhinolaryngology, Karaganda State Polyclinic, Seyfyllin street, Building 11a, Karaganda, Republic of Kazahstan; x461yom@mail.ru

6 "Andrija Štampar" School of Public Health, University of Zagreb, School of medicine, Rockefellerova 4, 10000, Zagreb, Croatia; pero.hrabac@mef.hr

7 Ivano-Frankivsk National Medical University, Galytska str, Kiev, Ukraine; popovychvasyl@gmail.ru

* Correspondence: DS danijela.stanfel@jgl.hr; KH kristina.hlaca@jgl.hr; Tel.: + 385 (0) 51546388

\begin{abstract}
:
The history of saline nasal irrigation (SNI) is indeed a long one, beginning from the ancient Ayurvedic practices and starting to gain a foothold in the west at the beginning of 20th century. Today, there is a growing number of papers covering effects of SNI from in vitro studies to randomized clinical trials and literature overviews. Based on the recommendations of most of the European and American professional associations, seawater, alone or in combination with other preparations, has its place in treatment of numerous conditions of the upper respiratory tract (URT), primarily in chronic (rhino)sinusitis, allergic rhinitis, acute URT infections and postoperative recovery. Additionally, taking into account its multiple mechanisms of action and mounting evidence from recent studies, locally applied seawater preparations may have an important role in prevention of viral and bacterial infections of the URT. Therefore, in this review we discuss results published in the past years focused on the seawater preparations and their use in clinical and everyday conditions, since such products are superior to saline, have an excellent safety profile and are recommended by most professional associations in the field of otorhinolaryngology.
\end{abstract}

Keywords: seawater; seawater preparation; Aqua Maris; nasal irrigation; upper respiratory track; otorhinolaryngology

\section{Introduction}

The use of water for prophylactic or therapeutic purposes, mostly in respiratory system, has been known since ancient times. In Yogic practices, different nasal cleansing techniques are used as part of a wider range of body cleansing procedures. Vedic texts describe several techniques called "neti" [1,2] with "jala neti" [3,4] corresponding to today's concept of nasal cavity irrigation. In the neti techniques, copperware was used for irrigation (to prevent contamination of the solution), the solution was heated to body temperature and an exact salt concentration in the preparation of solution was specified. This salt content and, consequently, the osmolality of the solution remained one of the most important parameters in nasal irrigation to the present day.

The osmolality of the commercial compositions of $\mathrm{NaCl}$ solution ranges from physiological $0.9 \%$ to hypertonic solutions with an osmolality of $3 \%$ [2]. Solutions with higher 
osmolality tend to induce side effects. The osmolality of the solution results not only from $\mathrm{NaCl}$ content but also from the other ions contained therein. Besides having effect on osmolality, the ions also show a number of effects on the biology and function of cells and tissues. This is especially important because many commercially available formulations, primarily these based on seawater, contain a number of ions other than $\mathrm{Na}^{+}$and $\mathrm{Cl}^{-}$and differ significantly from the galenic saline. In this review, the following parameters of these solutions will be discussed:

Composition of solution in context of differences between saline and solutions based on seawater,

Mechanism of action in nasal cavity and elsewhere, Safety and efficacy of use in different indications.

\section{Composition of saline/seawater preparations}

Unlike saline, which consists of $\mathrm{NaCl}$ dissolved in distilled water, in seawater there are four categories of constituents or solutes: major constituents, minor constituents, trace elements and gases. Average salinity of undiluted seawater is approximately $3.5 \%$ or 35 ppt (parts per thousand). Ninety-nine percent of seawater salinity is due to 6 major constituents: $\mathrm{Cl}-\mathrm{Na}^{+}, \mathrm{SO}_{4}{ }^{2-}, \mathrm{Mg}^{2+}, \mathrm{Ca}^{2+}$ and $\mathrm{K}^{+}$. Salinity is relatively uniform, with range of variation of 33-37 ppt in open ocean water, 37-38 in smaller bodies of seawater such as the Adriatic Sea to as much as $240 \mathrm{ppt}$ in the Dead Sea. This is why the source of water in seawater products is such an important factor.

One of the fundamental laws in oceanography, the Forchhammer principle or the principle of constant proportions, states that the relative proportions of the major constituents of seawater are constant, regardless of different salinities in different sea-water samples. $\mathrm{Cl}^{-}$accounts for $55 \%$ of the ions, followed by $\mathrm{Na}^{+}(30.6 \%), \mathrm{SO}_{4}{ }^{2-}(7.7 \%)$ and $\mathrm{Mg}^{2+}$ $(4 \%)$. Major constituents are also considered to be conservative, i.e., chemically non-reactive and thus stable in oceans and seas over the long periods of time. Besides major constituents, measured in ppt, seawater also contains a number of minor constituents (measured in ppm - parts per million) and trace elements, measured in ppb - parts per billion. However, the principles that apply to the major elements do not apply to the minor and trace elements. This means that many of these elements are biologically or chemically reactive and that their concentration can be dependent on biological activity and other factors, exhibiting significant local differences. Major constituents of seawater with salinity of $35 \mathrm{ppt}$ at the temperature of $25^{\circ} \mathrm{C}$ are shown in the Table 1.

Table 1. Major constituents of seawater $\left(\mathrm{mg} / \mathrm{dm}^{3}\right)$

\begin{tabular}{cccccc}
\hline Constituent & $\begin{array}{c}\text { Dittmar } \\
\mathbf{( 1 9 4 0 ) [ 5 ]}\end{array}$ & $\begin{array}{c}\text { Cox } \\
(\mathbf{1 9 6 6 ) [ 6 ]}\end{array}$ & $\begin{array}{c}\text { Riley } \\
\mathbf{( 1 9 6 7 ) [ 7 ]}\end{array}$ & $\begin{array}{c}\text { Millero } \\
\mathbf{( 1 9 9 6 ) [ 8 ]}\end{array}$ & $\begin{array}{c}\text { Štanfel } \\
\mathbf{( 2 0 0 6 ) [ 9 ]}\end{array}$ \\
\hline $\mathbf{C l}^{-}$ & 19805 & - & - & 19805 & 19763 \\
$\mathbf{N a}^{+}$ & 11015 & 11013 & 11037 & 11035 & $\mathbf{1 2 1 1 7}$ \\
$\mathbf{S O}^{2-}$ & 2764 & - & 2776 & 2764 & 2707 \\
$\mathbf{M g}^{2+}$ & 1327 & 1327 & 1322 & 1314 & $\mathbf{1 4 1 7}$ \\
$\mathbf{C a}^{2+}$ & 418 & 422 & 422 & 422 & $\mathbf{4 7 4}$ \\
$\mathbf{K}^{+}$ & 397 & 408 & 408 & 408 & $\mathbf{4 4 3}$ \\
$\mathbf{B r}$ & 67 & - & 69 & 69 & 63 \\
\hline
\end{tabular}

From Table 1 is evident that the cations (sodium, potassium, calcium and magnesium) determined in Adriatic Sea (Kvarner bay) by the ion-chromatography method are higher than cations obtained by various authors mentioned in the Table 1 . 
Table 2. Minor constituents of seawater[8]

\begin{tabular}{|c|c|c|c|}
\hline Constituent & $\mathrm{g} / \mathrm{kg}$ & AW & $\mathrm{mol} / \mathrm{kg} / \mathrm{H}_{2} \mathrm{O}$ \\
\hline $\mathrm{HCO}_{3}-$ & 0.10481 & 61.0168 & 0.0017803 \\
\hline $\mathbf{B}(\mathrm{OH})_{3}$ & 0.01944 & 61.8330 & 0.0003259 \\
\hline $\mathrm{CO}_{3}{ }^{2-}$ & 0.01434 & 60.0089 & 0.0002477 \\
\hline $\mathrm{Sr}^{2+}$ & 0.00795 & 87.6200 & 0.0000940 \\
\hline $\mathbf{B}(\mathrm{OH})_{4^{-}}$ & 0.00795 & 78.8404 & 0.0001045 \\
\hline $\mathrm{F}^{-}$ & 0.00130 & 18.9984 & 0.0000709 \\
\hline $\mathrm{CO}_{2}$ & 0.00042 & 44.0095 & \\
\hline $\mathrm{OH}^{-}$ & 0.00014 & 17.0073 & 0.0000085 \\
\hline Total (major+minor) & 35.16504 & & 1.1605659 \\
\hline $\mathrm{H}_{2} \mathrm{O}$ & 964.83496 & & 0.580283 \\
\hline
\end{tabular}

Another parameter of primary importance for seawater products is osmolality. To define the exact osmolality, an in-house study was performed to test the exact osmolality of seawater preparations as a function of seawater content in the final product. Results are shown in the Table 3.

Table 3. Osmolality of the product depending on the seawater content

\begin{tabular}{cc}
\hline Osmolality [mOsm/kg] & Seawater content [\%] \\
\hline 328 & 30.0 \\
318 & 29.0 \\
307 & 28.0 \\
298 & 27.0 \\
286 & 26.0 \\
277 & 25.0 \\
265 & 24.0 \\
260 & 23.0 \\
238 & 22.0 \\
235 & 21.0 \\
220 & 20.0 \\
\hline
\end{tabular}

The results show that seawater in concentrations of approximately $26 \%$ to $27 \%$, becomes hypertonic (considering plasma osmolality reference range of $285-295 \mathrm{mOsm} / \mathrm{kg}$ [10] and can exert a range of effects associated with hypertonic solutions.

\section{Mechanism of action}

The mechanism of action of saline/seawater solutions is based on two principles: physical and biological/physiological. The first principle is based on the physical (mechanical) effect of cleansing the nasal mucosa of the accumulated secretion and pathogens. The second principle depends on the effects of the ions on the physiology of the mucosal cells. In the Figure 1 we propose the chain of events following mucosal application of seawater preparations, resulting in a range of beneficiary effects. 


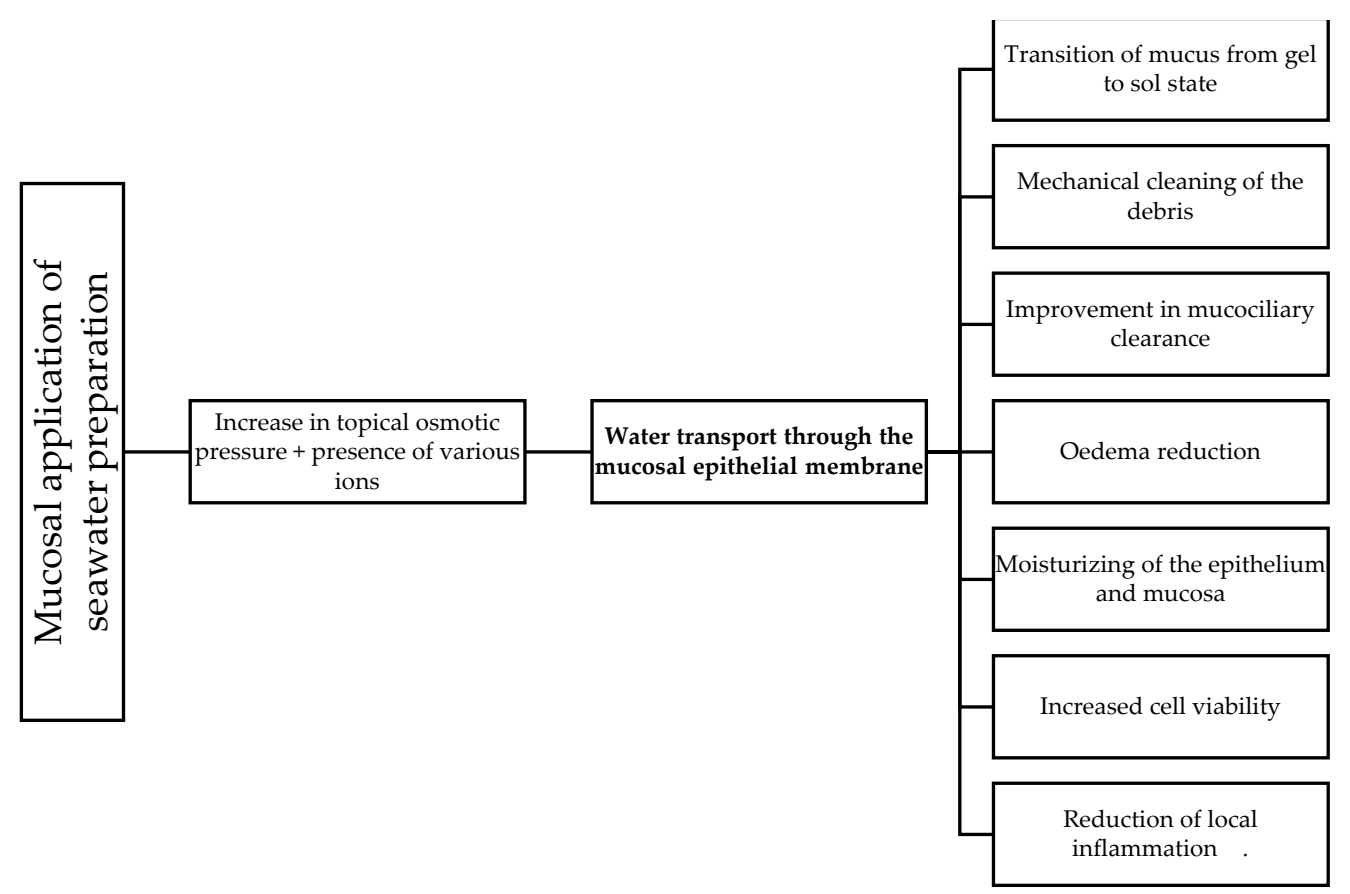

Figure 1. Proposed mechanism of action of seawater preparations locally applied to mucosa of the upper respiratory tract

Mechanism displayed in the Figure 1 above centres on the water transport through the mucosal epithelial membrane, provoked by the local application of hypertonic solution. Nasal mucosa is hydrated and moisturized by both local application of solution and influx of water through the membrane. Depending on the place of application, this leads to accumulation of liquid in the lumen and increased mucociliary clearance $[11,12]$. The mechanism has been proven both in vitro $[13,14]$ and in vivo $[15,16]$. Reduction of swelling (oedema) is seen in submucosal tissue, while the immediate effect of excess liquid in nasal lumen is mechanical cleaning of mucus, crusts and debris. Imminently following is the change of the state of the mucus from gel to sol [17]. Transition of mucus from gel to sol state greatly reduces the amount of energy needed by cilia to transport such mucus [18], significantly improving efficacy of the mucociliary transport. Additional ionic constituents of seawater show other effects such as increased cell viability and inflammation reduction (Figure 1 and Table 4).

Indeed, the efficacy of mucociliary transport might be one of the key mechanisms how saline/seawater solutions exert their positive effect on the nasal tissue. Mucociliary transit time (MTT; the time needed for a compound to be transported a certain distance within the respiratory system), is used to assess the efficacy of mucociliary clearance. Compared to healthy volunteers with mean MTT of $12.01+/-3.0$ minutes, this time is significantly prolonged in subjects with history of allergic rhinitis (15.5 +/- 3.5 minutes) and heavy smokers (16.5 +/- 5.0 minutes) [19]. Similarly, it has been shown that patients with a wide variety of diseases, ranging from septum deviations [20] to chronic sinusitis [21], have prolonged MTT and that the restoration of mucociliary clearance is of significant importance in treating the disease [22].

On the most basic level, MTT depends on the ciliary beat frequency (CBF). Wabnitz et al. used nasal sprays with $0.9 \%$ and $3.0 \%$ sodium chloride on eight healthy volunteers, having the mean baseline CBF of 9.6 Hz. While isotonic saline reduced the CBF first to 9.1 $\mathrm{Hz}$ (after 5 minutes) and $8.8 \mathrm{~Hz}$ (after one hour), use of $3.0 \%$ saline in-creased the CBF to $10.1 \mathrm{~Hz}$ before returning to near-baseline levels $(9.2 \mathrm{~Hz})$ at 60 minutes [23]. Similar results were seen by monitoring another parameter - saccharine clearance time, which decreased from median of $11.17 \mathrm{~min}$ to a median of 6.83 and 7.14 minutes after application of either 
isotonic or hypertonic saline, respectively [24]. These results, which show a beneficiary effect of hypertonic saline, but much smaller or completely absent effect of isotonic saline, are confirmed by other authors [25-28]. Same effects of hypertonic saline were shown for mucociliary clearance in asthmatic patients [29], subjects with cystic fibrosis [30-32], children with bronchiolitis [33] and healthy subjects [15]. On the molecular level, this effect seems to be based on the upregulation by the hypertonic saline of the CLC-3, a chloride channel that accounts for the transport of chloride ions in numerous tissues and plays a fundamental role in transepithelial salt and water movement [34].

Besides mentioned mechanism based on physical and osmotic effects of the solution, different ions contained in the seawater have a number of additional effects. These effects are displayed in the Table 4.

Table 4. Mechanism of action of other constituents seawater $[3,35]$

\begin{tabular}{ll}
\hline Constituent & Action \\
\hline & Promotes cell repair and limits inflammation by reducing the eicosanoid \\
& metabolism both at the level of the liberation of arachidonic acid and by \\
& direct inhibition of the 5-lipoxygenase enzyme, \\
& Inhibits exocytosis from permeabilized eosinophils, \\
& Reduces apoptosis of respiratory cells. \\
\hline & Acetylcholine and serotonin act as messengers, increasing calcium intake \\
& in ciliated cells and thus regulating ciliary beat frequency and synchroni- \\
& zation, \\
& Airflow promotes cell calcium intake and ciliary beat via shear-stress-in- \\
& duced mechanotransduction. \\
\hline $\mathbf{K}^{+}$ & Anti-inflammatory action, \\
& Promotes respiratory epithelium repair via the EGF/EGFR pathway. \\
\hline $\mathbf{H C O}_{3}{ }^{-}$ & $\begin{array}{l}\text { Reduces mucous viscosity by acting as a buffer, } \\
\text { Facilitates elimination by ciliary cells movement. }\end{array}$
\end{tabular}

Mentioned findings show that, besides the immediate positive effect of the mechanical cleaning of the mucosal surface, there is an additional and potentially more important positive effect exerted through facilitating the physiological function of mucociliary transport achieved by the saline solution of adequate osmolality. Additionally, other ions contained in the solution show a wide range of beneficiary physiological effects on cellular level.

\section{Aspects of saline/seawater in human use}

The Table 5 shows main safety and efficacy conclusions from clinical trials and invitro studies, performed over more than 20 years. We searched MEDLINE, Scopus, Web of Science and Cochrane databases to identify studies of interest. The aim was to identify as much as possible relevant (especially clinical) studies. To achieve this, we used a broad search strategy, including only basic keywords of "seawater" and "saline". For example, a MeSH search syntax was "Seawater"[Mesh] OR "Saline Solution"[Mesh] OR "Saline Solution, Hypertonic"[Mesh]. Considering that MeSH indexing takes some time, additional PubMed search with same keywords was performed for the studies published over the last three years. Additional studies were identified through Scopus and especially by following "Times Cited" links for the Web of Science results. After going through all the 
identified studies, we focused on the ones that, in our opinion, contribute most to the understanding of safety and efficacy aspects of saline/seawater use in human medicine. Covered are studies with both seawater and saline solutions in wide range of osmolalities and compositions. Safety and efficacy of these preparations will be shortly discussed here.

\subsection{Safety}

As it was already mentioned before, intranasal treatment with saline and sea-water preparations in form of either drops, spray, nebulizer or irrigation is considered to be very safe. Numerous studies, ranging from healthy individuals to infants and pregnant women, prove this point beyond a reasonable doubt. In Table 5, in more than 60 studies covering the period of last 23 years, general side effects are rare while serious ones virtually non-existent. Moreover, one must take into account the fact that in most of the studies subjects had at least one additional condition or diagnosis such as allergic rhinitis, rhinosinusitis, postoperative status, asthma, bronchiolitis etc. Most of these conditions require additional therapy which in itself could be the reason for side effect(s) ascribed to saline/seawater treatment. In the mentioned studies, in most cases adverse events are neither mentioned in the text of the papers or none were reported by study participants. In cases where adverse events have been mentioned, these were in most cases:

Burning feeling in nose and throat. Some studies report incidence of this adverse event to be rather high, so mild burning sensation was reported by majority (57\% [29]) subjects in study by Kumar et al. In the same study, moderate burning was much less pronounced, with only $19 \%$ subjects reporting this side effect. Also, the intensity of burning seemed to be correlated to osmolality of the preparation, with hypertonic preparations causing more adverse events. Other studies report similar rated of burning among their participants, so Shoseyov et al. [36] describe burning in 4 (of total of 34) paediatric subjects with chronic sinusitis, with 3 taking hypertonic saline and one taking isotonic preparation (note similar rate of adverse events between hypertonic and isotonic groups, as described in the previous study). However, there are studies where this rate is inverse [37]. Other studies mentioning burning as a side effect of saline/seawater therapy either fall within incidence boundaries described above [38,39] or discuss burning as a side effect not affecting subjects' participation in the study or study's outcome [27,40-42].

Other adverse events were rare and include nasal drainage [40,43], epistaxis [41,44,45], bitter taste in mouth [41], pain [46] and nose dryness [47].

\subsection{Efficacy}

The efficacy of saline/seawater solutions has been proven in numerous clinical trials and studies, most of which are listed in the Tables 5 and 6 . Efficacy has been proven in a variety of populations, from pregnant women and children to adults with a wide range of pathological conditions. Given that the attached list of publications speaks for itself, we will concentrate on presenting the essential facts about a few of the most important indications.

\subsubsection{Chronic sinusitis}

By definition, chronic rhinosinusitis (CRS) is an inflammation of the paranasal sinuses seen in several percent of both paediatric and adult population [48]. The diagnosis is based on the presence of at least 2 of 4 cardinal symptoms for at least 12 weeks and is confirmed by physical examination and (if necessary) additional radiological methods. Intranasal spray administration of corticosteroids is known to significantly improve symptoms, and a similar consensus exists for nasal saline irrigation. The use of oral antibiotics may be indicated in cases of acute exacerbations of the disease, although this was not corroborated in the recent Cochrane review on this topic [49]. Similar results have been described by a group of Russian authors in children [50]. 
Papers listed in Tables 5 and 6 strongly confirm these findings. In paediatric population Pham et al. [51] have shown that 6-week treatment is well tolerated in children and is useful both as a first-line treatment for CRS and as an effective measure reducing the need for surgery. Regarding tonicity, in another paediatric study, hypertonic solution was shown to be comparable to the isotonic in terms of safety, although the number of adverse events was higher in the hypertonic group [36].

Evidence of both safety and efficacy are, expectedly, more numerous in adult population. Subjects treated with nasal saline used less antibiotics compared to the control group [52] and hypertonic solution was reported as superior to the isotonic solution [5355]. Other hypertonic saline preparations such as the Dead Sea salt have also been proven as safe and effective in this indication [56]. While various application methods are used (mostly spray vs. low/large volume irrigation $[43,57]$, the safety profile remains highly favourable across the various studies.

\subsubsection{Allergic rhinitis}

Allergic rhinitis is an extremely common condition that is also commonly overlooked in the diagnostic process, resulting in significant public health effects. Also, although it is not a severe illness, allergic rhinitis can significantly complicate symptoms, diagnosis and clinical course of other diseases [58].

Saline and specifically seawater preparations have been shown to be effective [59] and safe [60] as both long-term [61] and short term [62] treatments and to reduce the need for other commonly used treatment options such as antihistamines in children [63,64] and pregnant women [65]. The same was proven for the use of nasal steroids [66,67] and systemic drugs [68].

\subsubsection{Other indications}

Besides the two major indications listed above, there are numerous studies in other indications, as well as in vitro studies [69] and those performed on healthy participants, with latter serving primary as the proof of concept for safety and efficacy of nasal saline and seawater treatments.

Different methods of saline penetration were tested using the Technetium-99 labelled solution, with douching being the method with best penetration in the maxillary sinus [70]. Positive effects of nasal irrigation were proven in healthy army conscripts [47], adult subjects $[14,23,71]$, and otherwise healthy subjects exposed to wood dust [44,72].

Regarding other indications, positive effects were described in paediatric patients with viral bronchiolitis [73], bronchiolitis in the intensive care unit [74], acute sinusitis [75], acute upper respiratory tract infections [76,77], chronic tonsilitis [78], cold and influenza [41]. Similar studies exist in adult subjects [79,80], including pregnant women [45]. Studies on postsurgical beneficiary effects of saline solutions [37,81], retrospective studies [82] as well as those based on questionnaires and surveys [42,83] seem to confirm all of the above mentioned effects.

\subsubsection{Place of saline/seawater preparations in COVID-19 pandemic}

Finally, although it is too early to speculate on whether the use of saline/seawater solutions has a place in preventing or reducing the symptoms of viral infections [84], a recent publication with people infected with coronavirus [80] suggests that this could be an interesting area of research in the near future. Also, there is a growing number of papers on this topic, suggesting potential positive effects of saline irrigations during the pandemic, both as preventive [86-88] and a treatment option [80]. A multidisciplinary group of Belgian authors in their recent paper [89] propose a detailed hypothesized mechanism of action of saline in coronavirus infections. The mechanism is quite similar to the one we 
propose in the present article including, among others, wetting properties to the local tissue, mucus gelling, and effects of the increased $\mathrm{NaCl}$ concentration on mucosa. Due to its effects described earlier in this paper and elsewhere [90], if used early and as an add-on therapy, locally applied saline/seawater solutions may represent an interesting and promising remedy for all viral infections, including SARS-CoV-2 [91].

Table 5. Overview of safety and efficacy conclusions from studies with saline and/or saltwater

\begin{tabular}{|c|c|c|c|c|}
\hline Study & Design & Subjects & Intervention & Safety conclusions \\
\hline $\begin{array}{l}\text { Holmstrom, } \\
1997 \text { [44] }\end{array}$ & $\begin{array}{l}\text { Cross-sec- } \\
\text { tional }\end{array}$ & $\begin{array}{l}45 \text { healthy adults } \\
\text { exposed to wood } \\
\text { dust }\end{array}$ & $\begin{array}{l}\text { Nasal lavage with } \\
\text { Rhinomer force 2, } \\
\text { four times a day, } \\
\text { every workday. }\end{array}$ & $\begin{array}{l}\text { One increase in allergic } \\
\text { symptoms (with concomi- } \\
\text { tant local steroid). One an- } \\
\text { terior epistaxis and one } \\
\text { throat irritation. }\end{array}$ \\
\hline $\begin{array}{l}\text { Shoseyov, } \\
1998 \text { [36] }\end{array}$ & $\mathrm{RCT}$ & $\begin{array}{l}34 \text { children with } \\
\text { chronic sinusitis }\end{array}$ & $\begin{array}{l}\text { Hypertonic }(3.5 \%) \\
\text { vs. isotonic saline, } \\
10 \text { drops, three } \\
\text { times daily for } 4 \\
\text { weeks. }\end{array}$ & $\begin{array}{l}\text { Three subjects in hyper- } \\
\text { tonic and one in isotonic } \\
\text { group left study because } \\
\text { of the burning feeling in } \\
\text { the nose and throat. }\end{array}$ \\
\hline
\end{tabular}

$\begin{array}{ll}\text { Rabone, 1999 } & \begin{array}{l}\text { Crossover } \\ \text { trial with 1- }\end{array} \\ \text { [72] } & \begin{array}{l}\text { year follow- } \\ \text { exposed to wood } \\ \text { dust }\end{array} \\ & \text { up }\end{array}$

\begin{tabular}{|c|c|c|}
\hline $\begin{array}{l}\text { Taccariello, } \\
1999 \text { [91] }\end{array}$ & $\mathrm{RCT}$ & $\begin{array}{l}40 \text { patients with } \\
\text { chronic rhinosi- } \\
\text { nusitis }\end{array}$ \\
\hline
\end{tabular}

\begin{tabular}{ll}
\hline Bachmann, & RCT \\
$2000[71]$ & $\begin{array}{l}40 \text { adults with pa- } \\
\text { ranasal sinus dis- } \\
\text { ease }\end{array}$
\end{tabular}

Gravity fed, home-

made unbuffered Generally safe, no notable isotonic saline for 2 adverse events. months.

Traditional alkaline nasal douche vs. a

sterile sea water No adverse effects menspray, in addition to tioned. their regular treatment.

\section{Other remarks}

\section{Isotonic Ems salt so-}

lution or isotonic

sodium chloride so- No adverse events menlution nasal irriga- tioned in either group. tion twice daily for 7 days.

\section{Nasal saline irriga-}

Heatley, 2001 Prospective [57] RCT 150 adults with tion with bulb sy- No significant adverse More than one-third of subchronic rhinosi- ringe or irrigation events; comparable effi- jects reported using less connusitis pot vs. placebo, cacy in all three groups. comitant medication. daily for 2 weeks.
Nasal saline irriga-

Rabago, 2002

[52]

RCT

76 adults with tion with $150 \mathrm{ml}$ acute or chronic daily per nostril for rhinosinusitis

6 months vs. no treatment.

\begin{tabular}{ll}
\hline Garavello, & Prospec- \\
$2003[63]$ & tive RCT
\end{tabular}

2003 [63] tive RCT

\section{Ten side effects of which 8}

were considered as "not Subjects treated with nasal sasignificant" and 2 as sig- line used statistically signifinificant but still were cantly less antibiotic com"highly satisfied" with the pared to control group. treatment.

20 children with Hypertonic saline No patients lost to follow allergic rhinitis in 10 subjects, no treatment in 10 subjects; $2.5 \mathrm{ml}$ in up and no adverse events reported.

Statistically significant decrease in use of oral antihistamines in hypertonic saline group.

each nostril three times daily for 6 weeks.

\begin{tabular}{|c|c|c|c|c|c|}
\hline $\begin{array}{l}\text { Lee, } 2003 \\
{[27]}\end{array}$ & $\begin{array}{l}\mathrm{RCT}, \\
\text { crossover }\end{array}$ & $\begin{array}{l}28 \text { healthy adult } \\
\text { subjects }\end{array}$ & $\begin{array}{l}\text { Hypertonic }(\mathrm{Si}- \\
\text { nomarin, } 3 \%) \text { or }\end{array}$ & $\begin{array}{l}\text { Conplainments of mild } \\
\text { prickling sensation after }\end{array}$ & $\begin{array}{l}\text { The effect of the hypertonic } \\
\text { solution is probably due to }\end{array}$ \\
\hline
\end{tabular}


isotonic saline. 10

sprays of both

preparations (on

different days) in

the same nostril.

nasal douching with hy-

pertonic seawater. changes in mucus viscoelastic properties.

\begin{tabular}{|c|c|c|c|c|}
\hline $\begin{array}{l}\text { Chkhartish- } \\
\text { vili, } 2004 \text { [68] }\end{array}$ & $\begin{array}{l}\text { Case-con- } \\
\text { trol open } \\
\text { clinical } \\
\text { trial }\end{array}$ & $\begin{array}{l}30 \text { children with } \\
\text { allergic rhinitis, } \\
\text { acute and } \\
\text { chronic bacterial } \\
\text { rhinosinusitis } \\
\text { and } 30 \text { children } \\
\text { in control group }\end{array}$ & $\begin{array}{l}\text { "Aqua Maris" sea- } \\
\text { water solution, ei- } \\
\text { ther irrigation or } 2 \\
\text { drops in nasal } \\
\text { cavity } 3 \text { times a } \\
\text { day from } 2 \text { to } 4 \\
\text { weeks. }\end{array}$ & $\begin{array}{l}\text { Nasal drops in children } \\
\text { up to } 2 \text { years of age were } \\
\text { well tolerated, with no } \\
\text { complication. No ad- } \\
\text { verse effects mentioned } \\
\text { for the irrigation group. }\end{array}$ \\
\hline $\begin{array}{l}\text { Tano, } 2004 \\
\text { [47] }\end{array}$ & $\begin{array}{l}\text { Prospec- } \\
\text { tive trial }\end{array}$ & $\begin{array}{l}108 \text { healthy } \\
\text { army conscripts }\end{array}$ & $\begin{array}{l}\text { 10-week nasal } \\
\text { spraying with } \\
\text { physiological } \\
\text { saline twice daily, } \\
\text { followed by a 10- } \\
\text { week period of } \\
\text { follow up. }\end{array}$ & $\begin{array}{l}\text { Two side effects of nose } \\
\text { dryness. }\end{array}$ \\
\hline
\end{tabular}

$\begin{array}{ll}\text { Wormald, } & \begin{array}{l}\text { Prospec- } \\ \text { tive, cross- }\end{array} \quad 12 \text { adult subjects } \\ \text { over study }\end{array}$

Wormald over study
Nasal irrigation with normal saline containing Technetium $99 \mathrm{~m}$ sulfur colloid
No adverse effects mentioned.

In subjects with bacterial rhinosinusitis time to relief of symptoms in Aqua Maris group was $7 \pm 3$. 2 days vs $10 \pm 2.4$ days in control group. In allergic rhinitis group Aqua Maris reduced the use of systemic drugs in 7 of 15 patients.

There was a mean of 0.7 episodes of upper respiratory tract infection during the spray period, compared with 1.0 episodes during the observation.

The nasal cavity was well irrigated three techniques (spray, nebulization, douching). Douching was significantly more effective in penetrating the maxillary sinus and frontal recess. The sphenoid and frontal sinuses were poorly irrigated by all three techniques.

Active-treatments were su-

Intranasal hyper-

tonic dead sea sa-

tive, randomized

Cordray, single2005 [92] blind, placebo-con-

line spray, intranasal aqueous triamcinolone trolled spray, placebo nasal saline spray for 7 days.

Hypertonic saline vs. no treatment; 3

Garavello, Prospec2005 [64] tive RCT

44 children with allergic rhinitis

sprays $(50 \mu \mathrm{l})$ in each nostril three times daily for 7 weeks.

Cells in the cul-
Cell cultures of fully differenti-

Kim, 2005 In vitro

[69] study
ated passage-2 normal human nasal epithelial cells tures were treated with pure water and with $0.3 \%$ (hypotonic), 0.9\% (isotonic) and 3\% (hypertonic) saline solutions.

Atomized nasal
douche, vs. nasal

Passali, 2005 [79]

\section{0 patients} RCT with acute viral rhinosinusitis lavages with isotonic sodium chloride solution.
Two subjects withdrew for adverse events (unknown group).

No adverse events in the treatment group. perior to placebo, especially corticosteroids. Dead Sea saline solution improved mucociliary clearance while $\mathrm{Mg}$ cation probably exerted antiinflammatory effects.

Statistically significant decrease in use of oral antihistamines in hypertonic saline group.

mRNA for major airway mucins analysis and morphologic analysis suggests that

In vitro study. pure water damaged epithelial cells and that only isotonic saline did not affect their morphology.

Atomized nasal douches sig-
No adverse effects mentioned. nificantly improved inspiratory and expiratory rhinomanometric resistance and 


\begin{tabular}{|c|c|c|c|c|c|}
\hline & & & & & $\begin{array}{l}\text { nasal volumes and normal- } \\
\text { ized mucociliary transport } \\
\text { time to a physiological level. }\end{array}$ \\
\hline $\begin{array}{l}\text { Wabnitz, } \\
2005[23]]\end{array}$ & $\begin{array}{l}\text { In-vitro } \\
\text { study }\end{array}$ & $\begin{array}{l}8 \text { healthy adult } \\
\text { subjects }\end{array}$ & $\begin{array}{l}\text { One application of } \\
\text { four sprays of hy- } \\
\text { pertonic ( } 3.0 \%) \text { sa- } \\
\text { line (one nostril) } \\
\text { and isotonic saline } \\
\text { (another nostril) in } \\
\text { all subjects. }\end{array}$ & $\begin{array}{l}\text { No adverse effects men- } \\
\text { tioned. }\end{array}$ & $\begin{array}{l}\text { Cell samples from subjects } \\
\text { receiving saline solutions. } \\
\text { The administration of hyper- } \\
\text { tonic saline results in a sig- } \\
\text { nificantly faster CBF } 5 \\
\text { minutes (but not } 60 \text { minutes) } \\
\text { after administration. }\end{array}$ \\
\hline $\begin{array}{l}\text { Friedman, } \\
2006[56]\end{array}$ & $\begin{array}{l}\text { Random- } \\
\text { ized, pro- } \\
\text { spective, } \\
\text { double- } \\
\text { blind } \\
\text { study }\end{array}$ & $\begin{array}{l}42 \text { adults seek- } \\
\text { ing treatment for } \\
\text { chronic rhinosi- } \\
\text { nusitis }\end{array}$ & $\begin{array}{l}\text { Nasal irrigation } \\
\text { using hypertonic } \\
\text { dead sea salt solu- } \\
\text { tion with hyper- } \\
\text { tonic saline. }\end{array}$ & $\begin{array}{l}\text { No adverse effects men- } \\
\text { tioned. }\end{array}$ & $\begin{array}{l}\text { Both groups had significant } \\
\text { improvement after treat- } \\
\text { ment. However, the dead sea } \\
\text { salt patients had signifi- } \\
\text { cantly better symptom relief } \\
\text { and showed improved } \\
\text { RQLQ(S) scores. }\end{array}$ \\
\hline $\begin{array}{l}\text { Rabago, } 2006 \\
{[40]}\end{array}$ & $\begin{array}{l}\text { Semi struc- } \\
\text { tured, in- } \\
\text { depth in- } \\
\text { terviews in } \\
\text { a 3-part, } \\
\text { multi- } \\
\text { method } \\
\text { study }\end{array}$ & $\begin{array}{l}28 \text { subjects with } \\
\text { frequent rhinosi- } \\
\text { nusitis and } \\
\text { chronic sinona- } \\
\text { sal symptoms. }\end{array}$ & $\begin{array}{l}\text { Hypertonic saline } \\
\text { nasal irrigation. }\end{array}$ & $\begin{array}{l}\text { Side effects as saline } \\
\text { drainage, nasal burning, } \\
\text { or irritation were noted } \\
\text { but not identified as im- } \\
\text { portant enough to stop } \\
\text { the treatment. }\end{array}$ & $\begin{array}{l}\text { This is a well-tolerated, inex- } \\
\text { pensive, effective, long-term } \\
\text { therapy that patients can use } \\
\text { at home with minimal train- } \\
\text { ing and follow-up. }\end{array}$ \\
\hline $\begin{array}{l}\text { Hauptman, } \\
2007 \text { [38] }\end{array}$ & RCT & $\begin{array}{l}80 \text { adult patients } \\
\text { with rhinosinus- } \\
\text { itis }\end{array}$ & $\begin{array}{l}1 \mathrm{ml} \text { of physiolog- } \\
\text { ical or hypertonic } \\
\text { saline to one nos- } \\
\text { tril. }\end{array}$ & $\begin{array}{l}\text { Increased nasal burn- } \\
\text { ing/irritation with hy- } \\
\text { pertonic compared to } \\
\text { physiological saline. }\end{array}$ & $\begin{array}{l}\text { Buffered physiological saline } \\
\text { significantly affected nasal } \\
\text { airway patency, whereas } \\
\text { buffered hypertonic saline } \\
\text { had no effect on nasal pa- } \\
\text { tency. }\end{array}$ \\
\hline $\begin{array}{l}\text { Kuzik, } 2007 \\
{[73]}\end{array}$ & $\begin{array}{l}\text { Prospec- } \\
\text { tive, ran- } \\
\text { domized, } \\
\text { double- } \\
\text { blinded, } \\
\text { controlled, } \\
\text { multicen- } \\
\text { ter trial }\end{array}$ & $\begin{array}{l}96 \text { infants with } \\
\text { viral bronchio- } \\
\text { litis }\end{array}$ & $\begin{array}{l}\text { Repeated doses of } \\
\text { nebulized } 3 \% \text { hy- } \\
\text { pertonic saline or } \\
0.9 \% \text { normal sa- } \\
\text { line, in addition to } \\
\text { routine therapy. }\end{array}$ & $\begin{array}{l}\text { All participants toler- } \\
\text { ated therapy without ap- } \\
\text { parent adverse effects } \\
\text { and were eventually dis- } \\
\text { charged after achieving } \\
\text { full recovery. }\end{array}$ & $\begin{array}{l}\text { Clinically relevant reduction } \\
\text { in length of stay to } 2.6+/-1.9 \\
\text { days in hypertonic saline } \\
\text { group, compared with } 3.5+/- \\
2.9 \text { days in the normal saline } \\
\text { group. }\end{array}$ \\
\hline $\begin{array}{l}\text { Pynnonen, } \\
2007 \text { [43] }\end{array}$ & $\begin{array}{l}\text { Prospec- } \\
\text { tive RCT }\end{array}$ & $\begin{array}{l}127 \text { adults with } \\
\text { chronic nasal } \\
\text { and sinus symp- } \\
\text { toms }\end{array}$ & $\begin{array}{l}\text { Irrigation with } \\
\text { large volume and } \\
\text { low positive pres- } \\
\text { sure or spray for } 8 \\
\text { weeks. }\end{array}$ & $\begin{array}{l}\text { Forty-one subjects re- } \\
\text { ported a total of } 67 \text { ad- } \\
\text { verse effects. Posttreat- } \\
\text { ment nasal drainage was } \\
\text { the most common ad- } \\
\text { verse effect }(n=14) \text { in } \\
\text { each group. }\end{array}$ & $\begin{array}{l}\text { Nasal irrigations performed } \\
\text { with large volume and deliv- } \\
\text { ered with low positive pres- } \\
\text { sure are more effective than } \\
\text { saline sprays for treatment of } \\
\text { chronic nasal and sinus } \\
\text { symptoms in a community- } \\
\text { based population. }\end{array}$ \\
\hline $\begin{array}{l}\text { Karpova, } \\
2008[78]\end{array}$ & $\begin{array}{l}\text { Open-label } \\
\text { parallel- } \\
\text { group trial }\end{array}$ & $\begin{array}{l}84 \text { children with } \\
\text { chronic tonsilitis }\end{array}$ & $\begin{array}{l}\text { Experimental } \\
\text { group with } 64 \\
\text { subjects using } \\
\text { Aqua Maris sea- } \\
\text { water solution } \\
\text { and control group } \\
\text { using furacilin so- } \\
\text { lution for 6-8 } \\
\text { courses of crypt } \\
\text { lavage. }\end{array}$ & $\begin{array}{l}\text { No adverse effects men- } \\
\text { tioned. }\end{array}$ & $\begin{array}{l}\text { Aqua Maris group showed } \\
\text { superior results in term of } \\
\text { odynophagia and dysphagia } \\
\text { severity and duration and } \\
\text { hyperaemia and infiltration } \\
\text { of the palatine arches. }\end{array}$ \\
\hline
\end{tabular}




\begin{tabular}{|c|c|c|c|c|c|}
\hline $\begin{array}{l}\text { Slapak, } 2008 \\
\text { [41] }\end{array}$ & $\begin{array}{l}\text { Prospec- } \\
\text { tive RCT } \\
\text { in parallel } \\
\text { groups }\end{array}$ & $\begin{array}{l}401 \text { children } \\
\text { with cold or in- } \\
\text { fluenza }\end{array}$ & $\begin{array}{l}\text { Nasal saline irri- } \\
\text { gation delivered } \\
\text { via jet flow, fine } \\
\text { spray or added to } \\
\text { standard medica- } \\
\text { tion vs. standard } \\
\text { medication alone. } \\
\text { Applied } 6 \text { times } \\
\text { daily in acute } \\
\text { phase and } 3 \text { times } \\
\text { daily for } 12 \text { weeks } \\
\text { after. }\end{array}$ & $\begin{array}{l}\text { At the second visit, only } \\
8.7 \% \text { patients recorded } \\
\text { nasal wash complaints, } \\
\text { and at the final visit, this } \\
\text { dropped to } 2.4 \% \text {. The } \\
\text { other reported com- } \\
\text { plaints were burning, } \\
\text { bitter taste and nose } \\
\text { bleeding. }\end{array}$ & $\begin{array}{l}\text { The saline treatment was } \\
\text { well tolerated. Most com- } \\
\text { plaints appeared in the me- } \\
\text { dium jet group and were as- } \\
\text { sociated with the stronger } \\
\text { flow of the wash. }\end{array}$ \\
\hline $\begin{array}{l}\text { Suslu, } 2009 \\
\text { [37] }\end{array}$ & $\begin{array}{l}\text { Prospec- } \\
\text { tive RCT }\end{array}$ & $\begin{array}{l}45 \text { adult subjects } \\
\text { after septoplasty }\end{array}$ & $\begin{array}{l}2.3 \% \text { buffered hy- } \\
\text { pertonic seawater, } \\
\text { buffered isotonic } \\
\text { saline, unbuffered } \\
\text { isotonic saline; ir- } \\
\text { rigation six times } \\
\text { daily for } 20 \text { days. }\end{array}$ & $\begin{array}{l}\text { No dropouts, no adverse } \\
\text { events mentioned. }\end{array}$ & $\begin{array}{l}\text { Buffered isotonic saline } \\
\text { group had worse nasal burn- } \\
\text { ing VAS score when com- } \\
\text { pared with both buffered hy- } \\
\text { pertonic and nonbuffered } \\
\text { isotonic saline solutions. }\end{array}$ \\
\hline $\begin{array}{l}\text { Ural, } 2009 \\
\text { [93] }\end{array}$ & $\begin{array}{l}\text { Observa- } \\
\text { tional }\end{array}$ & $\begin{array}{l}132 \text { adult sub- } \\
\text { jects }\end{array}$ & $\begin{array}{l}\text { Control, allergic } \\
\text { rhinitis, acute si- } \\
\text { nusitis and } \\
\text { chronic sinusitis } \\
\text { groups received } \\
\text { two daily doses of } \\
\text { hypertonic ( } 3 \% \text { ) or } \\
\text { isotonic nasal irri- } \\
\text { gation for } 10 \text { days. }\end{array}$ & $\begin{array}{l}\text { No patients lost to fol- } \\
\text { low up, and no serious } \\
\text { side effects or intoler- } \\
\text { ance necessitating cessa- } \\
\text { tion of irrigation re- } \\
\text { ported. }\end{array}$ & $\begin{array}{l}\text { Nasal irrigation with iso- } \\
\text { tonic or hypertonic saline } \\
\text { can improve mucociliary } \\
\text { clearance time in various na- } \\
\text { sal pathologies. }\end{array}$ \\
\hline $\begin{array}{l}\text { Gelardi, } 2009 \\
\text { [94] }\end{array}$ & $\begin{array}{l}\text { Random- } \\
\text { ized pilot } \\
\text { study }\end{array}$ & $\begin{array}{l}20 \text { adult subjects } \\
\text { with acute rhi- } \\
\text { nosinusitis }\end{array}$ & $\begin{array}{l}\text { A nasal syringe } \\
\text { (10 mL saline so- } \\
\text { lution, } 3 \text { times } \\
\text { daily for } 14 \text { days) } \\
\text { or the Lavonase } \\
\text { system ( } 250 \mathrm{~mL} \\
\text { saline solution sac, } \\
\text { twice daily for } 14 \\
\text { days). }\end{array}$ & $\begin{array}{l}\text { No adverse effects men- } \\
\text { tioned. }\end{array}$ & $\begin{array}{l}\text { Nasal irrigation with the La- } \\
\text { vonase system was found to } \\
\text { be more effective in reducing } \\
\text { symptoms and decreasing } \\
\text { nasal resistances. }\end{array}$ \\
\hline Li, 2009 [66] & $\mathrm{RCT}$ & $\begin{array}{l}26 \text { children with } \\
\text { allergic rhinitis }\end{array}$ & $\begin{array}{l}\text { Saline irrigation, } \\
\text { steroid therapy, } \\
\text { saline+steroid } \\
\text { therapy groups; } \\
\text { twice a day for } 8 \\
\text { weeks. }\end{array}$ & $\begin{array}{l}\text { No subjects lost to fol- } \\
\text { low up, no adverse } \\
\text { events in saline group. }\end{array}$ & $\begin{array}{l}\text { Saline use permits use of less } \\
\text { topical steroids in this indi- } \\
\text { cation. }\end{array}$ \\
\hline $\begin{array}{l}\text { Rabago, } 2009 \\
{[42]}\end{array}$ & $\begin{array}{l}\text { Electronic } \\
\text { question- } \\
\text { naire }\end{array}$ & $\begin{array}{l}330 \text { practicing } \\
\text { family physi- } \\
\text { cians in Wiscon- } \\
\text { sin, US }\end{array}$ & $\begin{array}{l}\text { Saline nasal irriga- } \\
\text { tion for upper res- } \\
\text { piratory condi- } \\
\text { tions. }\end{array}$ & $\begin{array}{l}\text { Respondents were not } \\
\text { queried directly about } \\
\text { perceived safety } \\
\text { profile of the treatment. }\end{array}$ & $\begin{array}{l}\text { Analysis showed that } 86.7 \% \\
\text { of respondents have used } \\
\text { the treatment as adjunctive } \\
\text { care for conditions including } \\
\text { chronic rhinosinusitis ( } 91 \%) \text {, } \\
\text { acute bacterial rhinosinusitis } \\
(67 \%) \text {, seasonal allergic rhi- } \\
\text { nitis (66\%), viral upper res- } \\
\text { piratory infection (59\%), } \\
\text { other allergic rhinitis (48\%), } \\
\text { irritant based congestion } \\
\text { (48\%) and rhinitis of preg- } \\
\text { nancy (17\%). }\end{array}$ \\
\hline
\end{tabular}




\begin{tabular}{|c|c|c|c|c|c|}
\hline $\begin{array}{l}\text { Cingi, } 2010 \\
{[59]}\end{array}$ & $\begin{array}{l}\text { Prospec- } \\
\text { tive }\end{array}$ & $\begin{array}{l}100 \text { adult sub- } \\
\text { jects with aller- } \\
\text { gic rhinitis }\end{array}$ & $\begin{array}{l}\text { Seawater gel nasal } \\
\text { spray in 4-hour in- } \\
\text { tervals, two } \\
\text { sprays per nostril, } \\
\text { from morning till } \\
\text { evening; for } 10 \\
\text { days. }\end{array}$ & $\begin{array}{l}\text { Gel was well-tolerated } \\
\text { with no side-effects oc- } \\
\text { curring. }\end{array}$ & $\begin{array}{l}\text { Statistically significantly de- } \\
\text { creased rating of nasal con- } \\
\text { gestion and discharge after } \\
\text { the } 10 \text { day regimen. }\end{array}$ \\
\hline $\begin{array}{l}\text { Culig, } 2010 \\
{[53]}\end{array}$ & RCT & $\begin{array}{l}60 \text { patients with } \\
\text { chronic rhinosi- } \\
\text { nusitis }\end{array}$ & $\begin{array}{l}\text { Isotonic vs hyper- } \\
\text { tonic seawater } \\
\text { spray solution, ap- } \\
\text { plied 3-6 times } \\
\text { daily. }\end{array}$ & $\begin{array}{l}\text { No adverse events were } \\
\text { observed. }\end{array}$ & $\begin{array}{l}\text { Hypertonic solution was sta- } \\
\text { tistically significant superior } \\
\text { to the isotonic for all symp- } \\
\text { toms. }\end{array}$ \\
\hline $\begin{array}{l}\text { Hermeling- } \\
\text { meier, } 2012 \\
{[60]}\end{array}$ & $\begin{array}{l}\text { Systematic } \\
\text { review and } \\
\text { meta-anal- } \\
\text { ysis }\end{array}$ & $\begin{array}{l}400 \text { subjects of } \\
\text { which } 86 \text { were } \\
\text { children/adoles- } \\
\text { cent and } 45 \text { were } \\
\text { pregnant }\end{array}$ & $\begin{array}{l}\text { Different treat- } \\
\text { ments. }\end{array}$ & $\begin{array}{l}\text { No adverse events men- } \\
\text { tioned, however not all } \\
\text { studies included safety } \\
\text { outcomes. }\end{array}$ & $\begin{array}{l}\text { Saline nasal irrigation using } \\
\text { isotonic solution can be rec- } \\
\text { ommended as complemen- } \\
\text { tary therapy in allergic rhini- } \\
\text { tis. }\end{array}$ \\
\hline $\begin{array}{l}\text { Satdhabudha, } \\
2012 \text { [95] }\end{array}$ & $\begin{array}{l}\text { Prospec- } \\
\text { tive RCT }\end{array}$ & $\begin{array}{l}81 \text { children with } \\
\text { allergic rhinitis }\end{array}$ & $\begin{array}{l}\text { Buffered hyper- } \\
\text { tonic }(1.25 \%) \text { sa- } \\
\text { line or isotonic sa- } \\
\text { line; nasal irriga- } \\
\text { tion } 2 \text { times daily } \\
\text { for } 4 \text { weeks. }\end{array}$ & $\begin{array}{l}\text { One subjects in each } \\
\text { study group experi- } \\
\text { enced nasal burning } \\
\text { during the first use. }\end{array}$ & $\begin{array}{l}\text { Satisfaction with nasal irri- } \\
\text { gation was comparable be- } \\
\text { tween groups. }\end{array}$ \\
\hline $\begin{array}{l}\text { Tantilipikorn, } \\
2012 \text { [39] }\end{array}$ & $\begin{array}{l}\text { Prospec- } \\
\text { tive RCT }\end{array}$ & $\begin{array}{l}50 \text { adult subjects } \\
\text { with chronic rhi- } \\
\text { nosinusitis after } \\
\text { endoscopic sur- } \\
\text { gery }\end{array}$ & $\begin{array}{l}\text { Dexpanthenol } \\
\text { (Mar Plus) vs. iso- } \\
\text { tonic saline nasal } \\
\text { sprays; } 4 \text { applica- } \\
\text { tions weekly on } \\
\text { 1st, 2nd, 4th and } \\
\text { 6th postoperative } \\
\text { weeks. }\end{array}$ & $\begin{array}{l}\text { Dropout rate was com- } \\
\text { parable between groups. } \\
\text { Three subjects in nasal } \\
\text { saline group reported } \\
\text { burning sensation. }\end{array}$ & $\begin{array}{l}\text { Product containing seawater } \\
\text { (Mar Plus) had better effi- } \\
\text { cacy and comparable safety } \\
\text { to nasal saline. }\end{array}$ \\
\hline $\begin{array}{l}\text { Kumar, } 2013 \\
{[54]}\end{array}$ & RCT & $\begin{array}{l}50 \text { subjects with } \\
\text { chronic sinusitis }\end{array}$ & $\begin{array}{l}\text { Hypertonic }(3.5 \%) \\
\text { or isotonic nasal } \\
\text { saline; } 10 \text { drops, } \\
\text { three times a day } \\
\text { in both nostrils, } \\
\text { for } 4 \text { weeks. }\end{array}$ & $\begin{array}{l}\text { None of the patients' } \\
\text { groups reported severe } \\
\text { burning sensation. Mild } \\
\text { burning sensation was } \\
\text { reported by } 14.3 \% \text { in iso- } \\
\text { tonic group and } 57.1 \% \text { in } \\
\text { hypertonic group. Mod- } \\
\text { erate burning sensation } \\
\text { was reported by } 19 \% \text { of } \\
\text { patients in hypertonic } \\
\text { group. }\end{array}$ & $\begin{array}{l}\text { Hypertonic saline nasal solu- } \\
\text { tion was more efficacious, } \\
\text { well tolerated and it im- } \\
\text { proved quality of life in pa- } \\
\text { tients. }\end{array}$ \\
\hline $\begin{array}{l}\text { Chen, } 2014 \\
{[62]}\end{array}$ & $\begin{array}{l}\text { Parallel } \\
\text { design } \\
\text { with } 3 \\
\text { groups }\end{array}$ & $\begin{array}{l}61 \text { children with } \\
\text { allergic rhinitis }\end{array}$ & $\begin{array}{l}\text { Nasal irrigation, } \\
\text { intranasal cortico- } \\
\text { steroid, and com- } \\
\text { bined treatment. }\end{array}$ & $\begin{array}{l}\text { No adverse events re- } \\
\text { ported by subjects. }\end{array}$ & $\begin{array}{l}\text { Nasal irrigation and de- } \\
\text { creased nasal corticosteroids } \\
\text { combination a significant } \\
\text { improvement in symptoms } \\
\text { and signs and a significant } \\
\text { decrease in the mean eosino- } \\
\text { phile count in nasal secre- } \\
\text { tions were observed at week } \\
12 .\end{array}$ \\
\hline $\begin{array}{l}\text { Low, } 2014 \\
{[81]}\end{array}$ & RCT & $\begin{array}{l}74 \text { adult subjects } \\
\text { after endoscopic } \\
\text { sinus surgery }\end{array}$ & $\begin{array}{l}\text { Normal saline, } \\
\text { Ringer's solution } \\
\text { and hypertonic sa- } \\
\text { line group. }\end{array}$ & $\begin{array}{l}\text { No adverse events men- } \\
\text { tioned. }\end{array}$ & $\begin{array}{l}\text { All groups showed an im- } \\
\text { provement with treatment in } \\
\text { SNOT-20 scores and VAS } \\
\text { scores, as well as endoscopic } \\
\text { evaluation of mucosa ap- } \\
\text { pearance over time but no } \\
\text { improvement of MCC. }\end{array}$ \\
\hline
\end{tabular}




$\begin{array}{lllll}\text { Marchisio, } & \begin{array}{l}\text { Question- } \\ \text { naire sent }\end{array} & \begin{array}{l}860 \text { primary care } \\ \text { pyediatricians }\end{array} & \begin{array}{l}\text { Nasal saline irri- } \\ \text { gation in pre- } \\ \text { school children. }\end{array} & \begin{array}{l}98.3 \% \text { of the participat- } \\ \text { ing physicians evaluated } \\ \text { the treatment as effec- } \\ \text { tive and safe. }\end{array}\end{array}$

\begin{tabular}{|c|c|c|c|c|}
\hline $\begin{array}{l}\text { Nguyen, } 2014 \\
{[67]}\end{array}$ & $\begin{array}{l}\text { Prospec- } \\
\text { tive, un- } \\
\text { blinded, } \\
\text { single-arm } \\
\text { pilot study }\end{array}$ & $\begin{array}{l}40 \text { subjects with } \\
\text { allergic rhinitis }\end{array}$ & $\begin{array}{l}\text { Large-volume } \\
\text { low-pressure sa- } \\
\text { line irrigation } \\
\text { twice daily for } 8 \\
\text { weeks to the on- } \\
\text { going regiment of } \\
\text { nasal corticoster- } \\
\text { oids. }\end{array}$ & $\begin{array}{l}\text { No adverse events re- } \\
\text { ported. }\end{array}$ \\
\hline $\begin{array}{l}\text { Pham, } 2014 \\
\text { [51] }\end{array}$ & $\begin{array}{l}\text { Retrospec- } \\
\text { tive cohort } \\
\text { study and } \\
\text { cross-sec- } \\
\text { tional sur- } \\
\text { vey }\end{array}$ & $\begin{array}{l}144 \text { children } \\
\text { with paediatric } \\
\text { chronic rhinosi- } \\
\text { nusitis }\end{array}$ & $\begin{array}{l}6 \text { weeks of once } \\
\text { daily nasal irriga- } \\
\text { tion. }\end{array}$ & $\begin{array}{l}\text { The results of a long- } \\
\text { term (median of } 48 \\
\text { months) follow-up in } 54 \\
\text { participants show treat- } \\
\text { ment as safe and well- } \\
\text { tolerated. }\end{array}$ \\
\hline $\begin{array}{l}\text { Stoelzel, } 2014 \\
\text { [96] }\end{array}$ & RCT & $\begin{array}{l}20 \text { adult subjects } \\
\text { with allergic rhi- } \\
\text { nitis }\end{array}$ & $\begin{array}{l}\text { Nasya/Prevalin (a } \\
\text { thixotropic nasal } \\
\text { gel) vs. isotonic } \\
\text { seawater nasal } \\
\text { spray; } 2 \text { sprays ( } 2 \\
\times 0.14 \mathrm{~mL} \text { ) into } \\
\text { each nostril. }\end{array}$ & $\begin{array}{l}\text { No adverse events re- } \\
\text { lated to the application } \\
\text { of the investigational } \\
\text { product were recorded. }\end{array}$ \\
\hline
\end{tabular}

\begin{tabular}{|c|c|c|}
\hline $\begin{array}{l}\text { Wang, } 2014 \\
{[75]}\end{array}$ & $\begin{array}{l}\text { Prospec- } \\
\text { tive, pla- } \\
\text { cebo-con- } \\
\text { trolled } \\
\text { RCT }\end{array}$ & $\begin{array}{l}60 \text { atopic chil- } \\
\text { dren with acute } \\
\text { sinusitis }\end{array}$ \\
\hline
\end{tabular}

\section{Standard treat-} ment (including systemic antibiotics, mucolytics and nasal decongestants) with nasal irrigation with normal saline vs. standard treatment alone.
About $40 \%$ of physicians expressed doubts about parental compliance mainly because of a certain difficulty in administration or the supposed invasiveness of the procedure.

Saline treatment significantly improved QOL, with no significant changes in nasal flows, pattern use of nasal steroids, or adverse events.

Nasal irrigation is effective as a first-line treatment for paediatric chronic rhinosinusitis and subsequent nasal symptoms, and reduces need for FESS and CT imaging.

There was no difference between the two treatment groups regarding the global assessment of tolerability provided by the investigators or by the subjects.

There were significant improvements in mean PRQLQ and nPEFR values for the irrigation compared to the

No significant side effects were recorded in the isotonic saline irrigation group. non-irrigation group. There was no significant difference in radiographic findings between the groups. The irrigation group recorded significant improvements in eye congestion, rhinorrhea, nasal itching, sneezing, and cough symptoms.

Hypertonic saline

Alvarez-Puebla, 2015 [97] CT
35 adults with asthma
(5\%, administered by nebulizer) or mannitol.
Treatments were well tolerated.
Mannitol and hypertonic saline behaved similarly at sputum induction.

No significant difference between saline and seawater groups in terms of nasal congestion but a significant difference between the control group and these two groups. Inhaled $2.8 \%$ hypertonic saline in normal subjects was associated with a short-lived acceleration of MC, predominately in the central airways.
Benn
$[14]$

$\begin{array}{ll}\text { RCT, open } & 12 \text { healthy } \\ \text { label, } & \begin{array}{l}12 \text { adults } \\ \text { cross-over }\end{array}\end{array}$

Hypertonic saline; No adverse events men$2.8 \% \mathrm{NaCl}, 4 \mathrm{ml}$. tioned. nasal drops $(2.3 \%)$

and control group

(no treatment).
No adverse events mentioned. 


\begin{tabular}{|c|c|c|c|c|c|}
\hline $\begin{array}{l}\text { Bonnomet, } \\
2016 \text { [13] }\end{array}$ & $\begin{array}{l}\text { Random- } \\
\text { ized, con- } \\
\text { trolled, } \\
\text { blinded, in } \\
\text { vitro } \\
\text { study }\end{array}$ & $\begin{array}{l}\text { Airway epithe- } \\
\text { lial cells ob- } \\
\text { tained from } 13 \\
\text { nasal polyps' ex- } \\
\text { plants }\end{array}$ & $\begin{array}{l}\text { Response (ciliary } \\
\text { beat frequency } \\
\text { and epithelial } \\
\text { wound repair } \\
\text { speed) of cells to } 3 \\
\text { isotonic nasal irri- } \\
\text { gation solutions: } \\
\text { normal saline } \\
0.9 \% \text {; non-diluted } \\
\text { seawater; and 30\% } \\
\text { diluted } \\
\text { seawater }\end{array}$ & In vitro study. & $\begin{array}{l}\text { Non-diluted seawater ob- } \\
\text { tains the best results on cili- } \\
\text { ary beat frequency and } \\
\text { wound repair speed vs nor- } \\
\text { mal saline showing a delete- } \\
\text { rious effect on epithelial cell } \\
\text { function. }\end{array}$ \\
\hline $\begin{array}{l}\text { Grasso, } 2018 \\
{[61]}\end{array}$ & $\begin{array}{l}\text { Prospec- } \\
\text { tive, con- } \\
\text { trolled } \\
\text { clinical } \\
\text { trial }\end{array}$ & $\begin{array}{l}60 \text { patients with } \\
\text { allergic rhinitis }\end{array}$ & $\begin{array}{l}\text { Daily, 5-month } \\
\text { treatment with } \\
\text { isotonic seawater } \\
\text { nasal spray en- } \\
\text { riched with man- } \\
\text { ganese (4 } \\
\text { puffs/day). }\end{array}$ & $\begin{array}{l}\text { No adverse events men- } \\
\text { tioned. }\end{array}$ & $\begin{array}{l}\text { The treatment significantly } \\
\text { decreased the number of epi- } \\
\text { sodes of acute allergic rhini- } \\
\text { tis and increased QOL with- } \\
\text { out the adverse effects of the } \\
\text { standard care therapy. }\end{array}$ \\
\hline $\begin{array}{l}\text { Bergmann, } \\
2019[45]\end{array}$ & $\begin{array}{l}\text { Uncon- } \\
\text { trolled, } \\
\text { prospec- } \\
\text { tive, longi- } \\
\text { tudinal CT }\end{array}$ & $\begin{array}{l}136 \text { patients } \\
\text { with disorders } \\
\text { of nose and pa- } \\
\text { ranasal sinuses } \\
\text { including } 11 \\
\text { pregnant } \\
\text { women and one } \\
\text { nursing mother }\end{array}$ & $\begin{array}{l}\text { Seawater nasal } \\
\text { spray }(2.7 \%)\end{array}$ & $\begin{array}{l}\text { One adverse event re- } \\
\text { ported (epistaxis). }\end{array}$ & $\begin{array}{l}\text { Over the study period (mean } \\
44 \text { days) statistically signifi- } \\
\text { cant reductions in } 10 \text { out of } \\
12 \text { symptoms was found. } \\
\text { Only for parameters "im- } \\
\text { pairment of taste" and "im- } \\
\text { pairment of food intake" no } \\
\text { significant change in symp- } \\
\text { toms was observed. }\end{array}$ \\
\hline $\begin{array}{l}\text { Bogomil'skij, } \\
2019 \text { [77] }\end{array}$ & $\begin{array}{l}\text { Uncon- } \\
\text { trolled, } \\
\text { prospec- } \\
\text { tive, longi- } \\
\text { tudinal CT }\end{array}$ & $\begin{array}{l}\text { Children aged 2- } \\
5 \text { years with } \\
\text { acute infectious } \\
\text { rhinitis (some } \\
\text { with viral } \\
\text { comorbidity) }\end{array}$ & Aqua Maris spray. & None reported. & $\begin{array}{l}\text { Rapid regression of symp- } \\
\text { toms such as nasal conges- } \\
\text { tion and snoring, a decrease } \\
\text { in the amount of nasal dis- } \\
\text { charge by the 3rd day from } \\
\text { the start of drug use and } \\
\text { normalization of the rhino- } \\
\text { scopic findings by 5-7th day } \\
\text { of treatment. }\end{array}$ \\
\hline $\begin{array}{l}\text { Stobbelaar, } \\
2019[74]\end{array}$ & $\begin{array}{l}\text { Retrospec- } \\
\text { tive study }\end{array}$ & $\begin{array}{l}104 \text { children up } \\
\text { to } 2 \text { years of age } \\
\text { with bronchio- } \\
\text { litis in intensive } \\
\text { care unit }\end{array}$ & $\begin{array}{l}\text { Nebulised hyper- } \\
\text { tonic saline. }\end{array}$ & $\begin{array}{l}\text { No adverse events men- } \\
\text { tioned. }\end{array}$ & $\begin{array}{l}\text { In respiratory syncytial virus } \\
\text { positive patients, the use of } \\
\text { nebulised hypertonic saline } \\
\text { was correlated with a de- } \\
\text { crease in the duration of res- } \\
\text { piratory support and the } \\
\text { length of stay by factors } 0.72 \\
\text { and } 0.81 \text {, respectively. }\end{array}$ \\
\hline $\begin{array}{l}\text { Craig, } 2019 \\
\text { [98] }\end{array}$ & $\begin{array}{l}\text { Prospec- } \\
\text { tive, ran- } \\
\text { domised, } \\
\text { controlled, } \\
\text { double- } \\
\text { blind, su- } \\
\text { periority } \\
\text { trial } \\
\end{array}$ & $\begin{array}{l}107 \text { children } \\
\text { aged } 6 \text { months } \\
\text { to } 5 \text { years } \\
\text { planned to have } \\
\text { a nasogastric } \\
\text { tube inserted in } \\
\text { emergency de- } \\
\text { partment }\end{array}$ & $\begin{array}{l}\text { Lidocaine and } \\
\text { phenylephrine na- } \\
\text { sal spray or } 0.9 \% \\
\text { sodium chloride } \\
\text { placebo nasal } \\
\text { spray, before na- } \\
\text { sogastric insertion }\end{array}$ & $\begin{array}{l}\text { Adverse effects occurred } \\
\text { in } 28 \% \text { of those who re- } \\
\text { ceived lidocaine and } \\
\text { phenylephrine and } 42 \% \\
\text { of those who received } \\
\text { placebo. }\end{array}$ & $\begin{array}{l}\text { Lidocaine and phe- } \\
\text { nylephrine nasal spray does } \\
\text { not reduce procedure-re- } \\
\text { lated distress associated with } \\
\text { nasogastric tube insertion in } \\
\text { young children compared } \\
\text { with saline. }\end{array}$ \\
\hline $\begin{array}{l}\text { Perić, } \\
2019[55]\end{array}$ & $\begin{array}{l}\text { Prospec- } \\
\text { tive, ran- } \\
\text { domized } \\
\text { study }\end{array}$ & $\begin{array}{l}30 \text { patients with } \\
\text { Aspirin-induced } \\
\text { chronic rhinosi- }\end{array}$ & $\begin{array}{l}\text { Hypertonic }(2.3 \% \\
\mathrm{NaCl}) \text { sea water } \\
\text { and isotonic } 0.9 \% \\
\mathrm{NaCl} \text {. }\end{array}$ & $\begin{array}{l}\text { Nasal discomforts were } \\
\text { detected in two patients } \\
\text { in hypertonic sea water }\end{array}$ & $\begin{array}{l}\text { Significantly lower total } \\
\text { symptom score during the } \\
7 \text { th, } 14 \text { th, } 21 \text { st and the } 28 \text { th } \\
\text { day, lower total endoscopic } \\
\text { score on the } 21 \text { st and } 28 \text { th }\end{array}$ \\
\hline
\end{tabular}


nusitis undergo-

ing endoscopic

sinus surgery group and in two pa-

tients in the isotonic

group.

\begin{abstract}
day, lower nasal obstruction,
facial pain/pressure, head-

ache and trouble sleeping, and lower nasal mucosal oedema, nasal secretion and nasal crusting in patients treated by hypertonic sea water.

The duration of illness was shorter in the intervention arm in the subset of patients infected with coronavirus (mean 5.6 vs. 8.1 days). The difference in the duration of blocked nose was -3.1 days, cough -3.3 days and hoarseness of voice -2.9 days in favour of hypertonic saline treatment.

Treatment did not compromise the integrity of the nasal epithelium in vitro but was effective for removal of foreign particles through MCC increase and for enhancing wound repair on nasal mucosa.
\end{abstract}

Seawater group was statistically significantly superior in terms of nasal congestion, nasal discharge, sleep quality and appetite, but not for cough and fatigue.

Table 6. Overview of review articles with saline and/or saltwater

\begin{tabular}{|c|c|c|c|c|}
\hline Study & Design & Indication(s) & Intervention(s) & Remarks \\
\hline $\begin{array}{l}\text { Papsin, } \\
2003 \\
{[101]}\end{array}$ & $\begin{array}{l}\text { Literature } \\
\text { review }\end{array}$ & $\begin{array}{l}\text { Rhinosinusitis, } \\
\text { allergic rhini- } \\
\text { tis, postopera- } \\
\text { tive irrigation, } \\
\text { common cold }\end{array}$ & $\begin{array}{l}\text { Nasal irrigation as an adjunct } \\
\text { treatment }\end{array}$ & $\begin{array}{l}\text { The procedure has been used safely by both } \\
\text { adults and children and has no documented se- } \\
\text { rious adverse effects. Trials indicate that pa- } \\
\text { tients treated with nasal irrigation rely less on } \\
\text { other medications and that some postsurgical } \\
\text { patients tend to require fewer visits to physi- } \\
\text { cians. Both effects are likely to have desirable } \\
\text { economic consequences for patients and the } \\
\text { health care system. }\end{array}$ \\
\hline $\begin{array}{l}\text { Brown, } \\
2004 \\
{[102]}\end{array}$ & $\begin{array}{l}\text { Literature } \\
\text { review }\end{array}$ & $\begin{array}{l}\text { (Chronic) si- } \\
\text { nusitis, sinona- } \\
\text { sal conditions, } \\
\text { rhinitis, post- } \\
\text { operative pa- } \\
\text { tients }\end{array}$ & $\begin{array}{l}\text { Isotonic and hyperthonic saline, } \\
\text { buffered/unbuffered solutions, } \\
\text { additives such as antibacterial or } \\
\text { antifungal agents, home recipes } \\
\text { vs. manufactured solutions }\end{array}$ & $\begin{array}{l}\text { Nasal irrigations are an important component } \\
\text { in the management of most sinonasal condi- } \\
\text { tions. Authors note on disparity of opinion } \\
\text { about the effects of irrigations on ciliary beat } \\
\text { frequency and mucociliary clearance and con- } \\
\text { troversy concerning irrigation tonicity and the } \\
\text { use of additives to the irrigating solution. }\end{array}$ \\
\hline
\end{tabular}




\begin{tabular}{|c|c|c|c|}
\hline Study & Design & Indication(s) & Intervention(s) \\
\hline $\begin{array}{l}\text { Harvey, } \\
2007 \\
{[103]}\end{array}$ & $\begin{array}{l}\text { Review } \\
\text { (Cochrane) }\end{array}$ & $\begin{array}{l}\text { Chronic sinusi- } \\
\text { tis }\end{array}$ & $\begin{array}{l}\text { Randomised controlled trials in } \\
\text { which saline was evaluated in } \\
\text { comparison with either no treat- } \\
\text { ment, a placebo, as an adjunct to } \\
\text { other treatments or against treat- } \\
\text { ments. The comparison of hyper- } \\
\text { tonic versus isotonic solutions. }\end{array}$ \\
\hline
\end{tabular}

Kassel, 2010 [104] Review (Cochrane) Upper respiratory tract infections
RCTs comparing topical nasal saline treatment to other intervenclinically diagnosed acute URTIs. tions in adults and children with
Zhang, 2008 [105]
Acute bronchiolitis in infants
Nebulized hypertonic saline alone or in conjunction with bronchodilators as an active intervention in infants with acute bronchiolitis.
Adappa, 2012 [106]
(Cochrane)

\begin{abstract}
Saline irrigations are well tolerated. Although minor side effects are common, the beneficial effect of saline appears to outweigh these drawbacks for the majority of patients. The use of topical saline could be included as a treatment adjunct for the symptoms of chronic rhinosinusitis.
\end{abstract}

Three RCTs (618 participants) were included. Most results showed no difference between nasal saline treatment and control. However, there was limited evidence of benefit with nasal saline irrigation in adults. Minor discomfort was not uncommon and $40 \%$ of babies did not tolerate nasal saline drops.

Current evidence suggests nebulized 3\% saline may significantly reduce the length of hospital stay among infants hospitalized with non-severe acute viral bronchiolitis and improve the clinical severity score in both outpatient and inpatient populations.

Physiologic saline irrigation is beneficial in the treatment of symptoms of CRS. Low-level evidence supports the effectiveness of topical antibiotics in the treatment of CRS. The use of topical antifungals is not supported by the majority of studies. Intranasal steroids are beneficial in the treatment of CRS with nasal polyposis. There is insufficient evidence to demonstrate a clear overall benefit for topical steroids in CRS without nasal polyposis.

The use of isotonic and hypertonic saline solutions is a valuable non-pharmacological treatment for nasal congestion in children, especially by improving mucociliary clearance and reducing the use of medications (antihistamines, decongestants, antibiotics, corticosteroids) during the treatment of URTIs. They are well tolerated and can be recommended for infants.

\begin{tabular}{lll}
\hline Bastier, & $\begin{array}{l}\text { Overview of } \\
\text { randomized } \\
\text { clinical tri- } \\
\text { als }\end{array}$ & $\begin{array}{l}\text { Different si- } \\
\text { nonasal pa- } \\
\text { thologies and } \\
\text { postoperative } \\
\text { care }\end{array}$ \\
\hline
\end{tabular}

Different treatments compared to nasal irrigation including rhinocorticoids, antihistamines, buffered, unbuffered, alkaline, hyperand isotonic saline Studies with follow-up period of at least three months comparing

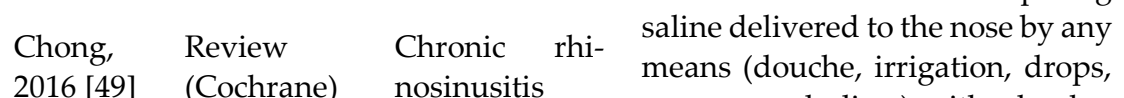
2016 [49] (Cochrane) nosinusitis $\begin{aligned} & \text { means (douche, irrigation, drops, } \\ & \text { spray or nebuliser) with placebo, }\end{aligned}$ no treatment or other pharmacological interventions

Large-volume low-pressure nasal irrigation using undiluted seawater seems, in the present state of knowledge, to be the most effective protocol.

The evidence suggests that there is no benefit of a low-volume nebulised saline spray over intranasal steroids. There is some benefit of daily, large-volume $(150 \mathrm{ml})$ saline irrigation with a hypertonic solution when compared with placebo.

Authors agree with the AAP guidelines regarding the use of nebulized hypertonic saline to reduce bronchiolitis scores and length of stay for infants with bronchiolitis who are expected to be hospitalized for more than 72 hours. 


\begin{tabular}{llll}
\hline \multicolumn{1}{c}{ Study } & Design & Indication(s) & \multicolumn{1}{c}{ Intervention(s) } \\
\hline $\begin{array}{l}\text { Madison, } \\
2016\end{array}$ & $\begin{array}{l}\text { Literature } \\
\text { review }\end{array}$ & $\begin{array}{l}\text { Allergic rhini- } \\
\text { tis in children }\end{array}$ & $\begin{array}{l}\text { Nasal saline irrigation vs. intrana- } \\
\text { sal corticosteroids }\end{array}$ \\
\hline 108$]$ & & &
\end{tabular}

\begin{tabular}{lll} 
& \multicolumn{2}{l}{ Systematic } \\
& search with \\
Kanjana- & Ovid MED- & Sinonasal dis- \\
wasee, & LINE, Sco- & eases, includ- \\
2018 & pus, Pub- & ing rhinitis and \\
{$[109]$} & Med and rhinosinusitis \\
& Google & \\
& Scholar
\end{tabular}

Hypertonic vs. isotonic saline

\section{Systematic} review and

Li, 2019 meta-analy-

Li, 2019 sis literature

[110] following the PRISMA

guidelines
Allergic rhini-

tis in children
Hypertonic saline nasal irrigation

Intranasal steroids are more effective than nasal saline alone to reduce symptoms of allergic rhinitis in children. However, combination therapy further improves symptom reduction. Nine studies (740 patients) were included. Hypertonic nasal irrigation brought greater benefits over isotonic in symptom reduction; however, no difference was shown in SNOT-20 improvement. Effects favouring hypertonic solution on symptoms were larger in patients with rhinitis compared with rhinosinusitis; patients under the age of 18 years; saline irrigation using high volume compared with low volume and saline irrigation with hypertonicity of $<3 \%$ and hypertonicity of 3\%-5\% compared with hypertonicity of $>5 \%$. No major adverse effects were reported.

Hypertonic saline treatment improved patients' nasal symptom scores and significantly lower rescue antihistamine use rate. Analyses comparing hypertonic with isotonic saline nasal irrigation better nasal symptom scores in hypertonic group, although the antihistamine use and adverse effect rates were similar between groups.

Saline nasal irrigation is recommended as an adjunct therapy for common colds/rhinosinusitis, chronic sinusitis, allergic rhinitis and after nasal surgery. It appears to be safe and generally well tolerated, even for children. The use of SNI has the potential to reduce the number of antibiotic prescriptions for acute and chronic sinus infections, and improve outcomes for patients.

King,

2019

[111]
Literature review with evidence for each of the indications
Chronic sinusi-

tis, allergic rhinitis, acute URTI
Saline solutions, dependent on the indication studied

\section{Conclusions}

Saline solutions eq Aqua Maris show numerous positive effects in clinical use in upper respiratory tract. These are mainly mechanical (cleaning of the mucosa) and related to osmolality (oedema reduction and moisturizing of the epithelium). In our paper we present a comprehensive body of evidence why sea-water is superior to saline for SNI in general as well as for the wide variety of clinical indications such as infectious diseases of the upper respiratory tract, allergic rhinitis, postoperative care etc. Due to its chemical constituents such as magnesium, calcium, potassium, bicarbonate and other ions, seawater shows a range of additional chemical effects from promoting cell repair and reducing inflammation to reducing viscosity of the mucus and increasing ciliary beat frequency. Numerous studies in URT patients, healthy volunteers, pregnant women, children and elderly prove exceptionally good safety profile of seawater preparations. Side effects are rare and consist mostly of burning feeling and nasal drainage, with serious adverse events practically non-existent.

To the best of our knowledge, a scientifically proven consensus on the exact mechanism of action of seawater in human upper respiratory tract does not exist. Therefore, and based on the comprehensive literature search, we propose a mechanism of action that considers all the different aspects of sea-water solution(s), from chemical composition to $\mathrm{pH}$ and tonicity. 


\begin{abstract}
Author Contributions: All authors have read and agreed to the published version of the manuscript. Acquired, analysed the data, D.Š.; conceptualization, investigation, writing-original draft preparation with review and editing, D.Š., K.H., P.H.; methodology and resources, D.Š., K.H., P.H, L.K., S.V.R., M.R.B., E.Y.R., R.T., V.I.P.
\end{abstract}

Funding: This research received no external funding

Acknowledgments: We wish to thank Marina Stanojević Večerina for initializing preparation of this systematic review, gathering the team, defining scope and coordination of activities, to Maša Safundžić Kučuk for her JGLs expertise in the development and production of seawater-based pharmaceutical products and Maša Margan Vunić for initial gathering of data.

Conflicts of Interest: The authors declare no conflict of interest.

1. Meera, S.; Vandana Rani, M.; Sreedhar, C.; Robin, D.T. A Review on the Therapeutic Effects of NetiKriya with Special Reference to JalaNeti. Journal of Ayurveda and integrative medicine 2020, 11, 185-189, doi:10.1016/J.JAIM.2018.06.006.

2. Khianey, R.; Oppenheimer, J. Is Nasal Saline Irrigation All It Is Cracked up to Be? Annals of allergy, asthma $\mathcal{E}$ immunology: official publication of the American College of Allergy, Asthma, \& Immunology 2012, 109, 20-28, doi:10.1016/J.ANAI.2012.04.019.

3. Principi, N.; Esposito, S. Nasal Irrigation: An Imprecisely Defined Medical Procedure. International journal of environmental research and public health 2017, 14, doi:10.3390/IJERPH14050516.

4. Ho, E.Y.; Cady, K.A.; Robles, J.S. A Case Study of the Neti Pot's Rise, Americanization, and Rupture as Integrative Medicine in U.S. Media Discourse. Health communication 2016, 31, 1181-1192, doi:10.1080/10410236.2015.1047145.

5. $\quad$ Lyman, J.; Fleming, R.H. Composition of Sea Water; J. mar. Res., 1940; Vol. 3(2);

6. Cox, R.A.; Culkin, F.; Riley, J.P.; Cox, R.A.; Culkin, F.; Riley, J.P. The Electrical Conductivity/Chlorinity Relationship in Natural Sea Water. DSRA 1967, 14, 203-220, doi:10.1016/0011-7471(67)90006-X.

7. Morris, A.W.; Riley, J.P. The Bromide/Chlorinity and Sulphate/Chlorinity Ratio in Sea Water. Deep Sea Research and Oceanographic Abstracts 1966, 13, 699-705, doi:10.1016/0011-7471(66)90601-2.

8. $\quad$ Millero, F.J. Chemical Oceanography; 4th ed.; CRC Press: Boca Raton, 2013;

9. Štanfel, D. Comparasion of Ion Chromatography with Other Methods in Sea Water Analysis of the Adriatic Coast, Zagreb, 2006.

10. Bhalla, A.; Sankaralingam, S.; Dundas, R.; Swaminathan, R.; Wolfe, C.D.A.; Rudd, A.G. Influence of Raised Plasma Osmolality on Clinical Outcome after Acute Stroke. Stroke 2000, 31, 2043-2048, doi:10.1161/01.STR.31.9.2043.

11. Daviskas, E.; Anderson, S.D.; Gonda, I.; Eberl, S.; Meikle, S.; Seale, J.P.; Bautovich, G. Inhalation of Hypertonic Saline Aerosol Enhances Mucociliary Clearance in Asthmatic and Healthy Subjects. The European respiratory journal 1996, 9, 725-732, doi:10.1183/09031936.96.09040725.

12. Trimble, A.T.; Whitney Brown, A.; Laube, B.L.; Lechtzin, N.; Zeman, K.L.; Wu, J.; Ceppe, A.; Waltz, D.; Bennett, W.D.; Donaldson, S.H. Hypertonic Saline Has a Prolonged Effect on Mucociliary Clearance in Adults with Cystic Fibrosis. Journal of cystic fibrosis: official journal of the European Cystic Fibrosis Society 2018, 17, 650-656, doi:10.1016/J.JCF.2018.01.001.

13. Bonnomet, A.; Luczka, E.; Coraux, C.; de Gabory, L. Non-Diluted Seawater Enhances Nasal Ciliary Beat Frequency and Wound Repair Speed Compared to Diluted Seawater and Normal Saline. International forum of allergy \& rhinology 2016, 6, 1062-1068, doi:10.1002/ALR.21782. 
14. Bennett, W.D.; Wu, J.; Fuller, F.; Balcazar, J.R.; Zeman, K.L.; Duckworth, H.; Donn, K.H.; O’Riordan, T.G.; Boucher, R.C.; Donaldson, S.H. Duration of Action of Hypertonic Saline on Mucociliary Clearance in the Normal Lung. Journal of applied physiology (Bethesda, Md.: 1985) 2015, 118, 1483-1490, doi:10.1152/JAPPLPHYSIOL.00404.2014.

15. Pavia, D.; Thomson, M.L.; Clarke, S.W. Enhanced Clearance of Secretions from the Human Lung after the Administration of Hypertonic Saline Aerosol. The American review of respiratory disease 1978, 117, 199-203, doi:10.1164/ARRD.1978.117.2.199.

16. Donaldson, S.H.; Bennett, W.D.; Zeman, K.L.; Knowles, M.R.; Tarran, R.; Boucher, R.C. Mucus Clearance and Lung Function in Cystic Fibrosis with Hypertonic Saline. The New England journal of medicine 2006, 354, 241-250, doi:10.1056/NEJMOA043891.

17. Quraishi, M.S.; Jones, N.S.; Mason, J. The Rheology of Nasal Mucus: A Review. Clinical otolaryngology and allied sciences 1998, 23, 403-413, doi:10.1046/J.1365-2273.1998.00172.X.

18. Sleigh, M.A. Ciliary Adaptations for the Propulsion of Mucus. Biorheology 1990, 27, 527-532, doi:10.3233/BIR1990-273-431.

19. Mahakit, P.; Pumhlrun, P. A Preliminary Study of Nasal Mucociliary Clearance in Smokers, Sinusitis and Allergic Rhinitis Patients. Asian Pacific Journal of Allergy and Immunology 1995, 13, 119-121.

20. Passàli, D.; Ferri, R.; Becchini, G.; Passàli, G.C.; Bellussi, L. Alterations of Nasal Mucociliary Transport in Patients with Hypertrophy of the Inferior Turbinates, Deviations of the Nasal Septum and Chronic Sinusitis. European archives of oto-rhino-laryngology: official journal of the European Federation of Oto-Rhino-Laryngological Societies (EUFOS) : affiliated with the German Society for Oto-Rhino-Laryngology - Head and Neck Surgery 1999, 256, 335-337, doi:10.1007/S004050050158.

21. Ohashi, Y.; Nakai, Y. Reduced Ciliary Action in Chronic Sinusitis. http://dx.doi.org/10.3109/00016488309105583 2009, 95, 3-9, doi:10.3109/00016488309105583.

22. Hafner, B.; Davris, S.; Riechelmann, H.; Mann, W.J.; Amedee, R.G. Endonasal Sinus Surgery Improves Mucociliary Transport in Severe Chronic Sinusitis. American journal of rhinology 1997, 11, 271-274, doi:10.2500/105065897781446612.

23. Wabnitz, D.A.M.; Wormald, P.J. A Blinded, Randomized, Controlled Study on the Effect of Buffered 0.9\% and 3\% Sodium Chloride Intranasal Sprays on Ciliary Beat Frequency. The Laryngoscope 2005, 115, 803-805, doi:10.1097/01.MLG.0000157284.93280.F5.

24. Homer, J.J.; England, R.J.; Wilde, A.D.; Harwood, G.R.J.; Stafford, N.D. The Effect of PH of Douching Solutions on Mucociliary Clearance. Clinical otolaryngology and allied sciences 1999, 24, 312-315, doi:10.1046/J.13652273.1999.00265.X.

25. Talbot, A.R.; Herr, T.M.; Parsons, D.S. Mucociliary Clearance and Buffered Hypertonic Saline Solution. The Laryngoscope 1997, 107, 500-503, doi:10.1097/00005537-199704000-00013.

26. Wen, Z.; Wu, C.; Cui, F.; Zhang, H.; Mei, B.; Shen, M. The Role of Osmolality in Saline Fluid Nebulization after Tracheostomy: Time for Changing? BMC Pulmonary Medicine 2016, 16, 1-10, doi:10.1186/S12890-016-0342X/FIGURES/5.

27. Lee, S.H.; Song, J.S.; Lee, S.H.; Hwang, S.J.; Lee, H.M. Effect of Hypertonic Seawater (Sinomarin(R)) on Mucociliary Clearance in Normal Subjects. Journal of Rhinology 2016, 10, 19-22. 
28. Keojampa, B.K.; Hoang Nguyen, M.; Ryan, M.W. Effects of Buffered Saline Solution on Nasal Mucociliary Clearance and Nasal Airway Patency. Otolaryngology--head and neck surgery : official journal of American Academy of Otolaryngology-Head and Neck Surgery 2004, 131, 679-682, doi:10.1016/J.OTOHNS.2004.05.026.

29. Daviskas, E.; Anderson, S.D.; Gonda, I.; Eberl, S.; Meikle, S.; Seale, J.P.; Bautovich, G. Inhalation of Hypertonic Saline Aerosol Enhances Mucociliary Clearance in Asthmatic and Healthy Subjects. The European respiratory journal 1996, 9, 725-732, doi:10.1183/09031936.96.09040725.

30. Eng, P.; Morton, J.; Douglass, J.; Riedler, J.; Wilson, J.; Robertson, C. Short-term Efficacy of Ultrasonically Nebulized Hypertonic Saline in Cystic Fibrosis. undefined 1996, doi:10.1002/(SICI)1099-0496(199602)21:2.

31. Stahl, M.; Wielpütz, M.O.; Ricklefs, I.; Dopfer, C.; Barth, S.; Schlegtendal, A.; Graeber, S.Y.; Sommerburg, O.; Diekmann, G.; Hüsing, J.; et al. Preventive Inhalation of Hypertonic Saline in Infants with Cystic Fibrosis (PRESIS). A Randomized, Double-Blind, Controlled Study. American journal of respiratory and critical care medicine 2019, 199, 1238-1248, doi:10.1164/RCCM.201807-1203OC.

32. Robinson, M.; Hemming, A.L.; Regnis, J.A.; Wong, A.G.; Bailey, D.L.; Bautovich, G.J.; King, M.; Bye, P.T.P. Effect of Increasing Doses of Hypertonic Saline on Mucociliary Clearance in Patients with Cystic Fibrosis. Thorax 1997, 52, 900-903, doi:10.1136/THX.52.10.900.

33. Baron, J.; El-Chaar, G. Hypertonic Saline for the Treatment of Bronchiolitis in Infants and Young Children: A Critical Review of the Literature. The journal of pediatric pharmacology and therapeutics : JPPT: the official journal of PPAG 2016, 21, 7-26, doi:10.5863/1551-6776-21.1.7.

34. Li, H. bin; Shi, J. bo; Cao, F.; Cheng, L.; Wen, W. ping; Xu, G. Hypertonic Saline Solution Stimulated CLC-3 Production in Primary Cultured Human Nasal Epithelial Cells. Auris, nasus, larynx 2008, 35, 47-51, doi:10.1016/J.ANL.2007.04.015.

35. Bastier, P.L.; Lechot, A.; Bordenave, L.; Durand, M.; de Gabory, L. Nasal Irrigation: From Empiricism to Evidence-Based Medicine. A Review. European annals of otorhinolaryngology, head and neck diseases 2015, 132, 281285, doi:10.1016/J.ANORL.2015.08.001.

36. Shoseyov, D.; Bibi, H.; Shai, P.; Shoseyov, N.; Shazberg, G.; Hurvitz, H. Treatment with Hypertonic Saline versus Normal Saline Nasal Wash of Pediatric Chronic Sinusitis. The Journal of allergy and clinical immunology 1998, 101, 602-605, doi:10.1016/S0091-6749(98)70166-6.

37. Süslü, N.; Bajin, M.D.; Süslü, A.E.; Öğretmenoğlu, O. Effects of Buffered 2.3\%, Buffered 0.9\%, and Non-Buffered 0.9\% Irrigation Solutions on Nasal Mucosa after Septoplasty. European archives of oto-rhino-laryngology : official journal of the European Federation of Oto-Rhino-Laryngological Societies (EUFOS) : affiliated with the German Society for Oto-Rhino-Laryngology - Head and Neck Surgery 2009, 266, 685-689, doi:10.1007/S00405-008-0807-5.

38. Hauptman, G.; Ryan, M.W. The Effect of Saline Solutions on Nasal Patency and Mucociliary Clearance in Rhinosinusitis Patients. Otolaryngology--head and neck surgery: official journal of American Academy of Otolaryngology-Head and Neck Surgery 2007, 137, 815-821, doi:10.1016/J.OTOHNS.2007.07.034.

39. Tantilipikorn, P.; Tunsuriyawong, P.; Jareoncharsri, P.; Bedavanija, A.; Assanasen, P.; Bunnag, C.; Metheetrairut, C. A Randomized, Prospective, Double-Blind Study of the Efficacy of Dexpanthenol Nasal Spray on the Postoperative Treatment of Patients with Chronic Rhinosinusitis after Endoscopic Sinus Surgery. J Med Assoc Thai 2012, 95, 58-63.

40. Rabago, D.; Barrett, B.; Marchand, L.; Maberry, R.; Mundt, M. Qualitative Aspects of Nasal Irrigation Use by Patients with Chronic Sinus Disease in a Multimethod Study. Annals of family medicine 2006, 4, 295-301, doi:10.1370/AFM.552. 
41. Šlapak, I.; Skoupá, J.; Strnad, P.; Horník, P. Efficacy of Isotonic Nasal Wash (Seawater) in the Treatment and Prevention of Rhinitis in Children. Archives of otolaryngology--head $\mathcal{E}$ neck surgery 2008, 134, 67-74, doi:10.1001/ARCHOTO.2007.19.

42. Rabago, D.; Zgierska, A.; Peppard, P.; Bamber, A. The Prescribing Patterns of Wisconsin Family Physicians Surrounding Saline Nasal Irrigation for Upper Respiratory Conditions. WMJ : official publication of the State Medical Society of Wisconsin 2009, 108, 145.

43. Pynnonen, M.A.; Mukerji, S.S.; Kim, H.M.; Adams, M.E.; Terrell, J.E. Nasal Saline for Chronic Sinonasal Symptoms: A Randomized Controlled Trial. Archives of otolaryngology-head E neck surgery 2007, 133, 1115-1120, doi:10.1001/ARCHOTOL.133.11.1115.

44. Holmström, M.; Rosén, G.; Wåhlander, L. Effect of Nasal Lavage on Nasal Symptoms and Physiology in Wood Industry Workers. Rhinology 1997, 35, 108-112.

45. Bergmann, C.; Müller, K.; Thieme, U.; Zeman, F.; Huppertz, G.; Koller, M.; Meiser, P. Real-World Data on the Use of Hypertonic Saline Nasal Spray in ENT Practice. SN Comprehensive Clinical Medicine 2019 1:5 2019, 1, 354361, doi:10.1007/S42399-019-0050-Y.

46. Chusakul, S.; Warathanasin, S.; Suksangpanya, N.; Phannaso, C.; Ruxrungtham, S.; Snidvongs, K.; Aeumjaturapat, S. Comparison of Buffered and Nonbuffered Nasal Saline Irrigations in Treating Allergic Rhinitis. The Laryngoscope 2013, 123, 53-56, doi:10.1002/LARY.23617.

47. Tano, L.; Tano, K. A Daily Nasal Spray with Saline Prevents Symptoms of Rhinitis. Acta oto-laryngologica 2004, 124, 1059-1062, doi:10.1080/00016480410017657.

48. Halawi, A.M.; Smith, S.S.; Chandra, R.K. Chronic Rhinosinusitis: Epidemiology and Cost. Allergy and asthma proceedings 2013, 34, 328-334, doi:10.2500/AAP.2013.34.3675.

49. Chong, L.Y.; Head, K.; Hopkins, C.; Philpott, C.; Glew, S.; Scadding, G.; Burton, M.J.; Schilder, A.G.M. Saline Irrigation for Chronic Rhinosinusitis. The Cochrane database of systematic reviews 2016, 4, doi:10.1002/14651858.CD011995.PUB2.

50. Radtsig, E.; Ermilova, N. v; Bogomilskiy, M.R. Nose Obstruction in Children: Causes and Methods of Treatment. Pediatrija. Žurnal IM. G.N. Speranskogo 2012, 91, 85-90.

51. Pham, V.; Sykes, K.; Wei, J. Long-Term Outcome of Once Daily Nasal Irrigation for the Treatment of Pediatric Chronic Rhinosinusitis. The Laryngoscope 2014, 124, 1000-1007, doi:10.1002/LARY.24224.

52. Rabago, D.; Zgierska, A.; Mundt, M.; Barrett, B.; Bobula, J.; Maberry, R. Efficacy of Daily Hypertonic Saline Nasal Irrigation among Patients with Sinusitis: A Randomized Controlled Trial. The Journal of family practice 2002, 51, 1049-1055.

53. Culig, J.; Leppée, M.; Vceva, A.; Djanic, D. Efficiency of Hypertonic and Isotonic Seawater Solutions in Chronic Rhinosinusitis. Med Glas (Zenica) 2010, 7, 116-123.

54. Kumar, R.A..; Viswanatha, B.; Krishna, N.; Jayanna, N.; Shetty, D.R. Efficacy of Hypertonic Saline and Normal Saline in the Treatment of Chronic Sinusitis. Int J Otolaryn Head Neck Surg 2013, 2, 90-96.

55. Perić, A.; Kovačević, S.V.; Barać, A.; Gaćeša, D.; Perić, A. v.; Jožin, S.M. Efficacy of Hypertonic (2.3\%) Sea Water in Patients with Aspirin-Induced Chronic Rhinosinusitis Following Endoscopic Sinus Surgery. Acta otolaryngologica 2019, 139, 529-535, doi:10.1080/00016489.2019.1605454.

56. Friedman, M.; Vidyasagar, R.; Joseph, N. A Randomized, Prospective, Double-Blind Study on the Efficacy of Dead Sea Salt Nasal Irrigations. The Laryngoscope 2006, 116, 878-882, doi:10.1097/01.MLG.0000216798.10007.76. 
57. Heatley, D.G.; McConnell, K.E.; Kille, T.L.; Leverson, G.E. Nasal Irrigation for the Alleviation of Sinonasal Symptoms. Otolaryngology--head and neck surgery: official journal of American Academy of Otolaryngology-Head and Neck Surgery 2001, 125, 44-48, doi:10.1067/MHN.2001.115909.

58. Greiner, A.N.; Hellings, P.W.; Rotiroti, G.; Scadding, G.K. Allergic Rhinitis. Lancet (London, England) 2011, 378, 2112-2122, doi:10.1016/S0140-6736(11)60130-X.

59. Cingi, C.; Halis Unlu, H.; Songu, M.; Yalcin, S.; Topcu, I.; Cakli, H.; Bal, C. Seawater Gel in Allergic Rhinitis: Entrapment Effect and Mucociliary Clearance Compared with Saline. Therapeutic advances in respiratory disease 2010, 4, 13-18, doi:10.1177/1753465809358190.

60. Hermelingmeier, K.E.; Weber, R.K.; Hellmich, M.; Heubach, C.P.; Mösges, R. Nasal Irrigation as an Adjunctive Treatment in Allergic Rhinitis: A Systematic Review and Meta-Analysis. American journal of rhinology $\mathcal{E}$ allergy 2012, 26, doi:10.2500/AJRA.2012.26.3787.

61. Grasso, M.; de Vincentiis, M.; Agolli, G.; Cilurzo, F.; Grasso, R. The Effectiveness of Long-Term Course of Sterimar Mn Nasal Spray for Treatment of the Recurrence Rates of Acute Allergic Rhinitis in Patients with Chronic Allergic Rhinitis. Drug design, development and therapy 2018, 12, 705-709, doi:10.2147/DDDT.S145173.

62. Chen, J.R.; Jin, L.; Li, X.Y. The Effectiveness of Nasal Saline Irrigation (Seawater) in Treatment of Allergic Rhinitis in Children. International journal of pediatric otorhinolaryngology 2014, 78, 1115-1118, doi:10.1016/J.IJPORL.2014.04.026.

63. Garavello, W.; Romagnoli, M.; Sordo, L.; Gaini, R.M.; di Berardino, C.; Angrisano, A. Hypersaline Nasal Irrigation in Children with Symptomatic Seasonal Allergic Rhinitis: A Randomized Study. Pediatric allergy and immunology: official publication of the European Society of Pediatric Allergy and Immunology 2003, 14, 140-143, doi:10.1034/J.1399-3038.2003.00021.X.

64. Garavello, W.; di Berardino, F.; Romagnoli, M.; Sambataro, G.; Gaini, R.M. Nasal Rinsing with Hypertonic Solution: An Adjunctive Treatment for Pediatric Seasonal Allergic Rhinoconjunctivitis. International archives of allergy and immunology 2005, 137, 310-314, doi:10.1159/000086462.

65. Garavello, W.; Somigliana, E.; Acaia, B.; Gaini, L.; Pignataro, L.; Gaini, R.M. Nasal Lavage in Pregnant Women with Seasonal Allergic Rhinitis: A Randomized Study. International archives of allergy and immunology 2010, 151, 137-141, doi:10.1159/000236003.

66. Li, H.; Sha, Q.; Zuo, K.; Jiang, H.; Cheng, L.; Shi, J.; Xu, G. Nasal Saline Irrigation Facilitates Control of Allergic Rhinitis by Topical Steroid in Children. ORL; journal for oto-rhino-laryngology and its related specialties 2009, 71, 5055, doi:10.1159/000178165.

67. Nguyen, S.A.; Psaltis, A.J.; Schlosser, R.J. Isotonic Saline Nasal Irrigation Is an Effective Adjunctive Therapy to Intranasal Corticosteroid Spray in Allergic Rhinitis. American journal of rhinology E allergy 2014, 28, 308-311, doi:10.2500/AJRA.2014.28.4066.

68. Chkhartishvili, E.; Zhorzholiani, L.; Karseladze, R. Nasal Disease Management by Using Aqua Maris Solution. Ann Biomed Res Edu 2004, 4, 186-187.

69. Kim, C.H.; Hyun Song, M.; Eun Ahn, Y.; Lee, J.G.; Yoon, J.H. Effect of Hypo-, Iso- and Hypertonic Saline Irrigation on Secretory Mucins and Morphology of Cultured Human Nasal Epithelial Cells. Acta oto-laryngologica 2005, 125, 1296-1300, doi:10.1080/00016480510012381.

70. Wormald, P.J.; Cain, T.; Oates, L.; Hawke, L.; Wong, I. A Comparative Study of Three Methods of Nasal Irrigation. The Laryngoscope 2004, 114, 2224-2227, doi:10.1097/01.MLG.0000149463.95950.C5. 
71. Bachmann, G.; Hommel, G.; Michel, O. Effect of Irrigation of the Nose with Isotonic Salt Solution on Adult Patients with Chronic Paranasal Sinus Disease. European archives of oto-rhino-laryngology: official journal of the European Federation of Oto-Rhino-Laryngological Societies (EUFOS) : affiliated with the German Society for Oto-RhinoLaryngology - Head and Neck Surgery 2000, 257, 537-541, doi:10.1007/S004050000271.

72. Rabone, S.J.; Saraswati, S.B. Acceptance and Effects of Nasal Lavage in Volunteer Woodworkers. Occupational medicine (Oxford, England) 1999, 49, 365-369, doi:10.1093/OCCMED/49.6.365.

73. Kuzik, B.A.; al Qadhi, S.A.; Kent, S.; Flavin, M.P.; Hopman, W.; Hotte, S.; Gander, S. Nebulized Hypertonic Saline in the Treatment of Viral Bronchiolitis in Infants. The Journal of pediatrics 2007, 151, doi:10.1016/J.JPEDS.2007.04.010.

74. Stobbelaar, K.; Kool, M.; de Kruijf, D.; van Hoorenbeeck, K.; Jorens, P.; de Dooy, J.; Verhulst, S. Nebulised Hypertonic Saline in Children with Bronchiolitis Admitted to the Paediatric Intensive Care Unit: A Retrospective Study. Journal of paediatrics and child health 2019, 55, 1125-1132, doi:10.1111/JPC.14371.

75. Wang, Y.H.; Ku, M.S.; Sun, H.L.; Lue, K.H. Efficacy of Nasal Irrigation in the Treatment of Acute Sinusitis in Atopic Children. Journal of microbiology, immunology, and infection = Wei mian yu gan ran za zhi 2014, 47, 63-69, doi:10.1016/J.JMII.2012.08.018.

76. Köksal, T.; Çizmeci, M.N.; Bozkaya, D.; Kanburoğlu, M.K.; Şahin, Ş.; Taş, T.; Yüksel, Ç.N.; Tatli, M.M. Comparison between the Use of Saline and Seawater for Nasal Obstruction in Children under 2 Years of Age with Acute Upper Respiratory Infection. Turkish journal of medical sciences 2016, 46, 1004-1013, doi:10.3906/SAG1507-18.

77. Bogomilskiy, M.R.; Radcig, E.; Radcig, A.N. Complex Treatment of Acute Infectious Rhinitis in Children. Pediatrija. Žurnal IM. G.N. Speranskogo 2019, 98, 120-123.

78. Karpova, E.P.; Feйzullaev, E.F. Experience in Using Laryngeal Aqua Maris Spray for the Local Treatment of Chronic Tonsillitis in Children. Vestnik otorinolaringologii 2008, 4, 85-86.

79. Passàli, D.; Damiani, V.; Passàli, F.M.; Passàli, G.C.; Bellussi, L. Atomized Nasal Douche vs Nasal Lavage in Acute Viral Rhinitis. Archives of otolaryngology--head $\mathcal{E}$ neck surgery 2005, 131, 788-790, doi:10.1001/ARCHOTOL.131.9.788.

80. Ramalingam, S.; Graham, C.; Dove, J.; Morrice, L.; Sheikh, A. Hypertonic Saline Nasal Irrigation and Gargling Should Be Considered as a Treatment Option for COVID-19. Journal of global health 2020, 10, doi:10.7189/JOGH.10.010332.

81. Low, T.H.; Woods, C.M.; Ullah, S.; Carney, A.S. A Double-Blind Randomized Controlled Trial of Normal Saline, Lactated Ringer's, and Hypertonic Saline Nasal Irrigation Solution after Endoscopic Sinus Surgery. American journal of rhinology \& allergy 2014, 28, 225-231, doi:10.2500/AJRA.2014.28.4031.

82. Rapiejko, P.; Jurkiewicz, D. [The Use of Hypertonic Sea-Water Solution in Patients after Surgery of the Nose and Paranasal Sinuses]. Otolaryngologia polska $=$ The Polish otolaryngology 2010, 64, 20-30, doi:10.1016/S00306657(10)70031-7.

83. Marchisio, P.; Picca, M.; Torretta, S.; Baggi, E.; Pasinato, A.; Bianchini, S.; Nazzari, E.; Esposito, S.; Principi, N. Nasal Saline Irrigation in Preschool Children: A Survey of Attitudes and Prescribing Habits of Primary Care Pediatricians Working in Northern Italy. Italian journal of pediatrics 2014, 40, doi:10.1186/1824-7288-40-47.

84. Baxter, A.L.; Schwartz, K.R.; Johnson, R.W.; Kuchinski, A.-M.; Swartout, K.M.; Rao, A.S.R.S.; Gibson, R.W.; Cherian, E.; Giller, T.; Boomer, H.; et al. Rapid Initiation of Nasal Saline Irrigation to Reduce Severity in High- 
Risk COVID+ Outpatients: A Randomized Clinical Trial Compared to a National Dataset Observational Arm. medRxiv 2021, 2021.08.16.21262044, doi:10.1101/2021.08.16.21262044.

85. Farrell, N.F.; Klatt-Cromwell, C.; Schneider, J.S. Benefits and Safety of Nasal Saline Irrigations in a PandemicWashing COVID-19 Away. JAMA otolaryngology-- head \& neck surgery 2020, 146, 787-788, doi:10.1001/JAMAOTO.2020.1622.

86. Casale, M.; Rinaldi, V.; Sabatino, L.; Moffa, A.; Ciccozzi, M. Could Nasal Irrigation and Oral Rinse Reduce the Risk for COVID-19 Infection? International journal of immunopathology and pharmacology 2020, 34, doi:10.1177/2058738420941757.

87. Panta, P.; Chatti, K.; Andhavarapu, A. Do Saline Water Gargling and Nasal Irrigation Confer Protection against COVID-19? Explore (New York, N.Y.) 2021, 17, 127-129, doi:10.1016/J.EXPLORE.2020.09.010.

88. Huijghebaert, S.; Hoste, L.; Vanham, G. Essentials in Saline Pharmacology for Nasal or Respiratory Hygiene in Times of COVID-19. European journal of clinical pharmacology 2021, 77, 1275-1293, doi:10.1007/S00228-021-031023.

89. Chatterjee, U.; Chakraborty, A.; Naskar, S.; Bandyapadhyay, B.; Shee, S. Efficacy of Normal Saline Nasal Spray and Gargle on SARS-CoV-2 for Prevention of COVID-19 Pneumonia. 2021, doi:10.21203/RS.3.RS-153598/V1.

90. Singh, S.; Sharma, N.; Singh, U.; Singh, T.; Mangal, D.; Singh, V. Nasopharyngeal Wash in Preventing and Treating Upper Respiratory Tract Infections: Could It Prevent COVID-19? Lung India : official organ of Indian Chest Society 2020, 37, 246-251, doi:10.4103/LUNGINDIA.LUNGINDIA_241_20.

91. Taccariello, M.; Parikh, A.; Darby, Y.; Scadding, G. Nasal Douching as a Valuable Adjunct in the Management of Chronic Rhinosinusitis. Rhinology 1999, 37, 29-32.

92. Cordray, S.; Harjo, J.B.; Miner, L. Comparison of Intranasal Hypertonic Dead Sea Saline Spray and Intranasal Aqueous Triamcinolone Spray in Seasonal Allergic Rhinitis. Ear, Nose and Throat Journal 2005, 84, 426-430, doi:10.1177/014556130508400713.

93. Ural, A.; Oktemer, T.K.; Kizil, Y.; Ileri, F.; Uslu, S. Impact of Isotonic and Hypertonic Saline Solutions on Mucociliary Activity in Various Nasal Pathologies: Clinical Study. The Journal of laryngology and otology 2009, 123, 517-521, doi:10.1017/S0022215108003964.

94. Gelardi, M.; Fiorella, M.L.; Gioacchino, M. di; Ciprandi, G. IMMUNE EFFECTS OF NICKEL View Project ARIA Italy ( Allergic Rhinitis and Its Impact on Asthma) View Project; 2009;

95. Satdhabudha, A.; Poachanukoon, O. Efficacy of Buffered Hypertonic Saline Nasal Irrigation in Children with Symptomatic Allergic Rhinitis: A Randomized Double-Blind Study. International journal of pediatric otorhinolaryngology 2012, 76, 583-588, doi:10.1016/J.JJPORL.2012.01.022.

96. Stoelzel, K.; Bothe, G.; Chong, P.W.; Lenarz, M. Safety and Efficacy of Nasya/Prevalin in Reducing Symptoms of Allergic Rhinitis. The clinical respiratory journal 2014, 8, 382-390, doi:10.1111/CRJ.12080.

97. Alvarez-Puebla, M.J.; Olaguibel, J.M.; Almudevar, E.; Echegoyen, A.A.; Vela, C.; de Esteban, B. Mannitol versus Hypertonic Saline: Safety and Efficacy of Mannitol and Hypertonic Saline in Sputum Induction and Bronchial Hyperreactivity Assessment. Chronic respiratory disease 2015, 12, 197-203, doi:10.1177/1479972315576144.

98. Craig, S.S.; Seith, R.W.; Cheek, J.A.; Wilson, K.; Egerton-Warburton, D.; Paul, E.; West, A. Lidocaine and Phenylephrine versus Saline Placebo Nasal Spray for the Pain and Distress of Nasogastric Tube Insertion in Young Children and Infants: A Randomised, Double-Blind, Controlled Trial. The Lancet. Child \& adolescent health 2019, 3, 391-397, doi:10.1016/S2352-4642(19)30058-6. 
99. Huang, S.; Constant, S.; de Servi, B.; Meloni, M.; Saaid, A.; Culig, J.; Bertini, M. Is a Diluted Seawater-Based Solution Safe and Effective on Human Nasal Epithelium? European archives of oto-rhino-laryngology : official journal of the European Federation of Oto-Rhino-Laryngological Societies (EUFOS) : affiliated with the German Society for OtoRhino-Laryngology - Head and Neck Surgery 2021, 278, 2837-2842, doi:10.1007/S00405-020-06527-1.

100. Jiang, M.; Chen, J.; Ding, Y.; Gan, C.; Hou, Y.; Lei, J.; Wan, M.; Li, X.; Xiao, Z. Efficacy and Safety of Sea SaltDerived Physiological Saline Nasal Spray as Add-On Therapy in Patients with Acute Upper Respiratory Infection: A Multicenter Retrospective Cohort Study. Medical science monitor: international medical journal of experimental and clinical research 2021, 27, doi:10.12659/MSM.929714.

101. Papsin, B.; McTavish, A. Saline Nasal Irrigation: Its Role as an Adjunct Treatment. Canadian Family Physician 2003, $49,168$.

102. Brown, C.L.; Graham, S.M. Nasal Irrigations: Good or Bad? Current opinion in otolaryngology E head and neck surgery 2004, 12, 9-13, doi:10.1097/00020840-200402000-00004.

103. Harvey, R.; Hannan, S.A.; Badia, L.; Scadding, G. Nasal Saline Irrigations for the Symptoms of Chronic Rhinosinusitis. The Cochrane database of systematic reviews 2007, doi:10.1002/14651858.CD006394.PUB2.

104. Kassel, J.C.; King, D.; Spurling, G.K. Saline Nasal Irrigation for Acute Upper Respiratory Tract Infections. The Cochrane database of systematic reviews 2010, doi:10.1002/14651858.CD006821.PUB2.

105. Zhang, L.; Mendoza-Sassi, R.A.; Wainwright, C.; Klassen, T.P. Nebulized Hypertonic Saline Solution for Acute Bronchiolitis in Infants. Cochrane Database of Systematic Reviews 2008, doi:10.1002/14651858.CD006458.PUB2/INFORMATION/EN.

106. Adappa, N.D.; Wei, C.C.; Palmer, J.N. Nasal Irrigation with or without Drugs: The Evidence. Current opinion in otolaryngology $\mathcal{E}$ head and neck surgery 2012, 20, 53-57, doi:10.1097/MOO.0B013E32834DFA80.

107. Chirico, G.; Quartarone, G.; Mallefet, P. Nasal Congestion in Infants and Children: A Literature Review on Efficacy and Safety of Non-Pharmacological Treatments. Minerva Pediatrica 2014, 66, 549-557.

108. Madison, S.; Brown, E.A.; Franklin, R.; Wickersham, E.A.; McCarthy, L.H. Nasal Saline or Intranasal Corticosteroids to Treat Allergic rhinitis in Children. The Journal of the Oklahoma State Medical Association 2016, $109,152$.

109. Kanjanawasee, D.; Seresirikachorn, K.; Chitsuthipakorn, W.; Snidvongs, K. Hypertonic Saline Versus Isotonic Saline Nasal Irrigation: Systematic Review and Meta-Analysis. American journal of rhinology $\mathcal{E}$ allergy 2018, 32, 269-279, doi:10.1177/1945892418773566.

110. Li, C.L.; Lin, H.C.; Lin, C.Y.; Hsu, T.F. Effectiveness of Hypertonic Saline Nasal Irrigation for Alleviating Allergic Rhinitis in Children: A Systematic Review and Meta-Analysis. Journal of clinical medicine 2019, 8, doi:10.3390/JCM8010064.

111. King, D. What Role for Saline Nasal Irrigation? Drug and therapeutics bulletin 2019, 57, 56-59, doi:10.1136/DTB.2018.000023. 